\title{
A Simulation Method for the Computation of the Effective P-Wave Velocity in Heterogeneous Rocks
}

\author{
Ángel Javier Omella*1, Julen Alvarez-Aramberri ${ }^{2,3}$, Magdalena \\ Strugaru $^{2}$, Vincent Darrigrand ${ }^{4}$, David Pardo ${ }^{1,2,5}$, Héctor \\ González $^{6}$, and Carlos Santos ${ }^{6}$ \\ ${ }^{1}$ Universidad del País Vasco (UPV/EHU), Leioa, Spain. \\ ${ }^{2}$ Basque Center for Applied Mathematics (BCAM), Bilbao, Spain. \\ ${ }^{3}$ Columbia University, Lamont-Doherty Earth Observatory, \\ Palisades, New York, US. \\ ${ }^{4}$ CERFACS. Toulouse, France. \\ ${ }^{5}$ Ikerbasque, Bilbao, Spain. \\ ${ }^{6}$ Repsol Tech Lab, Madrid, Spain.
}

\begin{abstract}
We propose a set of numerical methods for the computation of the frequency-dependent effective primary wave velocity of heterogeneous rocks. We assume the rocks' internal microstructure is given by microcomputed tomography images. In the low/medium frequency regime, we propose to solve the acoustic equation in the frequency domain by a Finite Element Method (FEM). We employ a Perfectly Matched Layer to truncate the computational domain and we show the need to repeat the domain a sufficient number of times to obtain accurate results. To make this problem computationally tractable, we equip the FEM with nonfitting meshes and we precompute multiple blocks of the stiffness matrix. In the high-frequency range, we solve the eikonal equation with a Fast Marching Method. Numerical results confirm the validity of the proposed methods and illustrate the effect of density, porosity, and the size and distribution of the pores on the effective compressional wave velocity.
\end{abstract}

*ajaome@gmail.com 


\section{Introduction}

In geophysical applications, it is of paramount importance to characterize the effective velocity of an elastic wave traveling through the Earth's subsurface. This information enables to identify gas saturated rocks as well as the porosity and other key petrophysical properties of the subsurface, and it is critical for an initial assessment of a reservoir.

According to the frequency of operation, we distinguish three different measurement acquisition systems for the characterization of subsurface elastic properties: (a) seismic data (typically below $100 \mathrm{~Hz}$ ), (b) logging sonic measurements (from $2 \mathrm{kHz}$ up to $300 \mathrm{kHz}$ ), and (c) core samples (analyzed at $300 \mathrm{kHz}-2 \mathrm{MHz}$ ). After proper interpretation of the results via advanced numerical methods (see [9, 39, 51]) we obtain an Earth map with its macroscale velocities. Unfortunately, maps obtained with measurements acquired at dissimilar frequencies are essentially different since a given heterogeneous rock exhibits dispair effective velocities at different frequencies; see e.g., [12, 30, 32, 36, 37, 59, 60. This undesired phenomena occurs due to the nature of wave propagation in heterogeneous rocks 42 .

Biot's theory [8] describes the wave propagation phenomena over a porous rock. However, it ignores the microscopic level and assumes that all the minerals of the rock have the same bulk and shear moduli [40. Moreover, the application of this theory using elastic or poro-elastic models is computationally expensive. In here, we focus on estimating the effective P-wave velocity using the acoustic equation and without incurring in a prohibitive computational cost. Hence, we are interested in the first arriving compressional $(\mathrm{P})$ wave that propagates along heterogeneous rocks in the excitation direction. Specifically, we focus only in the first wavefront travel-time, neglecting errors in the amplitude values. Thus, although compressional-to-shear (P-to-S) and S-to-P energy conversions may occur, they may be ignored, and an acoustic approximation of elastic waves is valid in this case, overcoming the limitations exposed in [11.

We need to analyze the structure of a given porous rock and estimate its effective $\mathrm{P}$-wave velocities at different frequencies. With X-ray micro-CT technology (see [19] for a review), it is possible to characterize the internal structure of rock samples at pore scale, identifying with precision the spatial distribution of its constituents. The current resolution of micro-CT available at laboratories of oil companies provides a set of around 1500 images of 1200 x 1200 pixels per core sample. This allows us to identify the material of each voxel in three dimensions (3D) with sub-pore resolution.

After producing a micro-CT scan, we need to analyze its effective velocities at different frequencies. The high-quality requirements for the cores destined to ultrasonic testing makes expensive the laboratory measurements and often can only be reliably estimated at high frequencies (above $500 \mathrm{kHz}$ ) to avoid undesired reflections. Some geometrical recommendations to properly analyze cores for ultrasonic testing are available in [2].

On the other hand, the use of relatively simple bounds and "averaging" formulas is extended in the industry for computing the stiffness tensor and 
the effective velocity from the single properties of each constituent. Some averaging formulas are the so-called Voigt 62 and Reus [50 bounds, VoigtReuss-Hill average [31, Hashin-Shtrikman bounds [28, 29], Kuster-Toksöz formulation for low-porosity rocks [34, Backus-average [3, 6, 54, Gassmann's Equation [7, 18, 25], Wyllie time average [20, 53, 63, Raymer's [49] and Gardner's 24 relations. These analytical and semi-analytical formulas omit the specific spatial distribution of the rock constituents. In a layered 1D media perpendicular to the direction of propagation, the Backus average is exact in the low-frequency limit, while the Wyllie time-average drives the high-frequency regime. However, when it comes to realistic two-dimensional (2D) and threedimensional (3D) heterogeneous rocks, all aforementioned theories fall short to provide accurate effective velocities at different frequencies.

There also exist multiple effective media theories, see [17, 23, 44]. For example, Differential Effective Medium (DEM) 43] considers different pore-type inclusions. Models for cracked medium are available in [13, 16, 22, 27, 33, 61]. There also exist a plethora of multi-scale methods for heterogeneous materials (see e.g., [10, 15, 21] or 26, 38, for a review). While some multi-scale methods are difficult to implement for industrial applications [1, others are unsuitable for high-frequency computations where it is crucial to determine the location of the micro-constituents in the rock at pore-scale. To the best of our knowledge, existing homogenization techniques often fail to solve the problem in the wide range of frequencies, as needed by our application.

Herin, we propose a set of simple-to-implement numerical methods that are capable of handling different frequencies and are suitable for the oil industry needs. These methods produce accurate approximations of the first $\mathrm{P}$-wave arrival corresponding to heterogeneous rocks.

The numerical results presented throughout this work exhibit the need of repeating the rock several times in the low-frequency regime. This increases the size of the problem (information of thousands of millions of pixels in 3D problems with possibly different material properties) and the computational cost, becoming the problem computationally untractable via conventional numerical methods. Moreover, since this is a wave propagation problem over an open domain, truncating the computational domain becomes a great challenge that should be carefully treated to preserve the accuracy of the homogenized velocities. Finally, it is also challenging to estimate effective velocities from a given (simulated) wave propagation solution.

In the low-medium frequency range, we solve the frequency-dependent wave equation by a FEM. To avoid undesired reflections from the domain boundary, we implement a Perfectly Matched Layer (PML). At low frequencies, we observe that this PML adversely affects the results. Thus, we need to extend virtually the original domain by repeating it multiple times. This increases dramatically the problem size. Traditional FEM employ fitting meshes in which material properties are continuous within each element. The large number of pixels attached to the necessity of the rock repetition leads to a prohibitive computational cost when using this technique, especially in $2 \mathrm{D}$ and $3 \mathrm{D}$. To overcome this challenge, we propose the use of non-fitting meshes 14, which 
reduces dramatically the number of degrees of freedom when the domain is large. However, this technique needs to be carefully employed to preserve the accuracy of the results. The repetition of the original domain allows to precompute blocks in FMMthe stiffness matrix in order to reduce the integration and assembling time.

After simulating the complex-valued pressure field in the frequency domain, we apply two methods to estimate the effective compressional wave velocity: (a) Prony's method [46], which provides an accurate value of effective compressional wave velocity at low frequencies, but it diverges at large ones, and (b) counting the number of wavelengths, which provides valid (although sometimes exhibiting low accuracy) approximations of the effective velocities across the entire frequency spectrum.

In the high-frequency limit, we solve the eikonal equation to approximate the travel time of the wave by a Fast Marching Method (FMM) [56]. Results in 1D heterogeneous rocks match with those obtained by the application of the Wyllie Time-Average 63. This confirms the accuracy of our proposed method. Thus, we apply it to estimate the high-frequency P-wave velocity limit in $2 \mathrm{D}$ and 3D problems, where analytical methods are unavailable for general porous rocks.

The remainder of the manuscript is organized as follows. Section2 2 introduces the theoretical bounds in layered media for the P-wave velocity. Section 3 describes the acoustic formulation in time and frequency. Then, we explain the physical need to repeat the rock, and we introduce two techniques to accelerate the computations: non-fitting meshes and precomputed block matrix. After that, we describe two methods to obtain the effective compressional wave velocity. To conclude this section, we analyze the high-frequency range, in which we propose to solve the eikonal equation to estimate the effective velocity. Section 4 focuses on the numerical results. The first four considered experiments analyze numerically the techniques presented for low-medium frequencies; experiments 5-8 show the influence of the main physical quantities (density, porosity, the size of the pore, and the distribution in the sizes of the pores) in the effective velocities. Experiment 9 shows the effective velocity profile computed in non-periodic vertical transversely isotropic (VTI) rocks. Experiment 10 describes the necessity to extend the domain in the perpendicular dimension of the $\mathrm{P}$-wave propagation direction. Experiment 11 illustrates the method scalability in higher dimensions. Experiment 12 shows the results for horizontal transversely isotropic (HTI) rocks. Experiment 13 checks the validity of the FMM in a synthetic rock. Finally, experiment 14 describes numerical results obtained from images of real rocks acquired in the laboratory via microCT. The last section is devoted to conclusions.

\section{Theoretical Bounds in Layered Media}

We consider a heterogeneous rock composed of various materials. Backus [3] showed that a stratified medium composed of VTI layers behaves like a 
homogeneous VTI media in the long-wavelength limit. To determine it, we search for "a global" fourth-order stiffness tensor $\mathcal{C}_{\text {eff }}$ that allows to treat the multi-material body as a homogenized effective one. The Backus homogenized $c_{i j k \ell}^{*}$ components of $\mathcal{C}_{e f f}$ are defined by relations among the components of $\mathcal{C}$, and by using the volumetric weighted average defined as $\{\cdot\}$.

Attending to this homogenization, the vertically propagating $P$-wave velocity 35 is:

$$
v_{\mathrm{P}, \mathrm{v}}=\sqrt{\frac{c_{3333}^{*}}{\rho^{*}}},
$$

where $c_{3333}^{*}=\left\{c_{3333}^{-1}\right\}^{-1}$, and $\rho^{*}=\{\rho\}$ is the volumetric weighted average of the densities.

Given a VTI layered rock, we consider known some information about each $i$-constituent (or layer): the volume fraction $\left(\phi_{i}\right)$, and some physical properties as density $\left(\rho_{i}\right)$ and compressional wave velocity $\left(v_{i}\right)$. Following (1), we use the Backus average to obtain the effective $\mathrm{P}$-wave velocity $v_{\text {eff }}^{B}$ :

$$
v_{\mathrm{P}, \mathrm{v}}=v_{e f f}^{B}=\sqrt{\frac{c_{3333}^{*}}{\sum_{i=1}^{n} \phi_{i} \rho_{i}}}, \text { where } \frac{1}{\mathrm{c}_{3333}^{*}}=\sum_{\mathrm{i}=1}^{\mathrm{n}} \frac{\phi_{\mathrm{i}}}{\rho_{\mathrm{i}} \mathrm{v}_{\mathrm{i}}^{2}} .
$$

Wyllie time-average 63 describes the effective velocity in high-frequency regimes $v_{\text {eff }}^{T A}$ as:

$$
\frac{1}{v_{e f f}^{T A}}=\sum_{i=1}^{n} \frac{\phi_{i}}{v_{i}} .
$$

Figure 1 shows a schematic representation of the effective velocity with respect to the frequency. Specifically, we represent (a) the Backus average zone, where the effective velocity tends to $v_{\text {eff }}^{B}$; (b) the time-average zone where the effective velocity approximates $v_{\text {eff }}^{T A}$; and (c), the transition zone that occurs between the two aforementioned zones. The frequency limits in this transition zone are unclear and an analytical expression for the effective velocity is unknown. Indeed, within this transition zone, the behavior of the effective velocity with respect to the frequency is often not even monotonic 60 .

\section{Mathematical Model and Solution Method}

In this section, we consider a rock with heterogeneous density and we first focus on the low/medium frequency range. We introduce the mathematical model, followed by the FEM adopted to solve it. Next, we discuss the need to repeat virtually the original domain several times until it becomes at least $n$-wavelengths long, where $n$ is typically between two and four. To make these problems computationally tractable, we consider a FEM with non-fitting meshes and precomputed stiffness matrices. Then, we use the solution obtained with FEM to evaluate the effective velocity via two different techniques, both based 




Figure 1: Schematic representation of the three frequency regimes and the effective velocity profile with respect to frequency. $\lambda$ is the frequency, and $d$ represents the pore size average.

on the post-processing of the solution sampled at multiple points. To conclude this section, we focus on the high-frequency regime. Specifically, we introduce the eikonal equation and a Fast Marching Method (FMM) to solve it.

\subsection{Acoustics}

When the density varies with position [5], the strong formulation of the acoustic problem in a fluid domain $\Omega$ is governed by the time-dependent wave equation,

$$
\nabla \cdot\left(\frac{1}{\rho} \nabla \hat{u}\right)-\frac{1}{\rho c^{2}} \frac{\partial^{2} \hat{u}}{\partial t^{2}}=\hat{f} \quad \text { in } \Omega,
$$

where $\hat{u} \equiv \hat{u}(\boldsymbol{x}, t)$ is the sound pressure, $c \equiv c(\boldsymbol{x})$ is the propagation velocity of the sound wave in the fluid, $\rho \equiv \rho(\boldsymbol{x})$ is the material density, and $\hat{f} \equiv \hat{f}(\boldsymbol{x}, t)$ is the volumetric stationary source term. In order to have unicity in the solution, we consider a Sommerfeld radiation condition [55, 58, which imposes proper decay conditions at infinity:

$$
\lim _{r \rightarrow \infty}\left(r\left(\frac{\partial \hat{u}}{\partial r}+\frac{1}{c} \frac{\partial t}{\partial \hat{u}}\right)\right)=0,
$$

where $r$ denotes the radial component in the spherical coordinate system. 


\subsection{Low-Medium Frequencies Analysis}

\subsubsection{Mathematical Model}

Applying a Fourier transform in time to equation (4), we obtain the following hyperbolic partial differential equation in the frequency domain:

$$
\nabla \cdot\left(\frac{1}{\rho(\boldsymbol{x})} \nabla u(\boldsymbol{x}, \omega)\right)+\frac{k^{2}(\boldsymbol{x})}{\rho(\boldsymbol{x})} u(\boldsymbol{x}, \omega)=f(\boldsymbol{x}, \omega) \quad \text { in } \Omega,
$$

where $u$ and $f$ stand for the Fourier transforms in time of $\hat{u}$ and $\hat{f}$, respectively, and $k(\boldsymbol{x})=\frac{\omega}{c(\boldsymbol{x})}$ is the medium wavenumber at angular frequency $\omega$. In addition, the Sommerfield radiation condition in spherical coordinates becomes:

$$
\lim _{r \rightarrow \infty}\left(r\left(\frac{\partial u}{\partial r}-i k u\right)\right)=0
$$

\subsubsection{Finite Element Method (FEM)}

To solve equation (6) using a FEM, we first introduce a polygonal domain $\Omega_{h}$, over which we generate the mesh $\mathcal{T}_{h}=\{\boldsymbol{K}\}$. Then, we define the space of finite elements $V_{h}:=\left\{v_{h} \in H^{1}\left(\Omega_{h}\right)\left|v_{h}\right|_{K} \in \mathcal{P}_{p}(K), \forall K \in \mathcal{T}_{h}\right.$, and $\left.\left.v_{h}\right|_{\partial \Omega_{h}}=0\right\}$, where $\mathcal{P}_{p}(K)$ is the space of polynomial functions of order $p$. We then rewrite 6 as a discrete variational problem:

$$
\left\{\begin{array}{l}
\text { Find } u_{h} \in V_{h}, \text { such that: } \\
-\int_{\Omega_{h}} \frac{1}{\rho} \nabla u_{h} \cdot \nabla v_{h}+\int_{\Omega_{h}} \frac{k^{2}}{\rho} u_{h} v_{h}=\int_{\Omega_{h}} f v_{h}, \\
\text { for all } v_{h} \in V_{h},
\end{array}\right.
$$

where $u_{h}$ is the approximated solution of $u(\boldsymbol{x}, \omega)$ that appears by solving a system of linear equations.

We employ a PML 4] to truncate the computational domain and impose homogeneous Dirichlet boundary conditions on its outer part. This ensures a proper Sommerfeld radiation condition and a lack of reflections from the boundary.

Repetition Strategy. We first execute simulations at low frequencies inside the Backus regime- and we observe incorrect values of the effective velocity. This occurs because the PML avoids boundary reflections, while in this application we seek for the homogenized velocities when the rock is repeated multiple times in the subsurface. This problem also affects laboratory experiments and partially explains the difficulties encountered when analyzing rocks at low frequencies. To overcome it in numerical simulations, we repeat the rock domain sample several times until we obtain a computational domain in which the effective velocity is unaffected by a surrounding (homogeneous slower) media. Experiments 3, 4 and 10 in Section 4 illustrate this strategy. 
Non-fitting Meshes. The standard FEM employs fitting meshes to assemble the bilinear form associated with the material. In particular, it assumes that density $\rho$ and wavenumber $k$ are sufficiently smooth within each cell $K$. However, this assumption makes the resulting simulator prohibitively expensive in our case since it would force us to consider thousands of millions of elements. For the sake of simplicity, we focus on the diffusive term of (8), and we write for a single element $S$ :

$$
\int_{S} \frac{k^{2}}{\rho} \phi_{j} \phi_{i}=\operatorname{area}(S) \frac{k_{S}^{2}}{\rho_{S}} \int_{\hat{S}} \hat{\phi}_{j} \hat{\phi}_{i}
$$

where $\phi_{i}$ is the $i$-th shape function associated with the element $S$, and $\hat{\phi}_{i}$ is the $i$-th shape function corresponding to the reference element $\hat{S}$.

The key ingredient in non-fitting meshes [14 is to group two or more cells, with possibly different material properties, into a single macro-element $K$ (see Figure 2p. Let $s$ be the number of sub-cells (elements) grouped into a macroelement: $K=\bigcup_{l=1}^{s} S_{l}$, where $S_{l}, l=1, \ldots, s$. Assuming that the material properties are constant inside each sub-cell $S_{l}$, we rewrite equation $(9)$ for nonfitting meshes as:

$$
\int_{K} \frac{k^{2}}{\rho} \phi_{j} \phi_{i}=\sum_{l=1}^{s} \int_{S_{l}} \frac{k^{2}}{\rho} \phi_{j} \phi_{i}=\sum_{l=1}^{s} \operatorname{area}(S) \frac{k_{K, l}^{2}}{\rho_{K, l}} \int_{\hat{S}_{l}} \hat{\phi}_{j} \hat{\phi}_{i},
$$

where $\hat{S}_{l}$ is the sub-cell associated with the reference macro-element $\hat{K}$, and as before, $\phi_{i}$ is the $i$-th shape function associated with the macro-element $K$, and $\hat{\phi}_{i}$ is the $i$-th shape function corresponding to the reference macro-element $\hat{K}$. Notice that we employ a standard FEM with non-fitting meshes. We do not employ multiscale basis functions.



(a) Fitting mesh



(b) Non-fitting mesh

Figure 2: Sketch of the shape functions (blue lines) and the numbering of elements over a formation with six pixels. Black and white pixels represent materials with a different density and velocity.

By using non-fitting meshes, we select larger macro-elements and perform exact integration. This reduces the number of unknowns in the finite element model and the corresponding computational requirements (time and memory). For more details about this technique, we refer the reader to [14].

Precomputed Matrix. We consider an original master domain $\Omega_{0}$ discretized by the master mesh $\mathcal{T}_{0}=\left\{K_{1}^{0}, K_{2}^{0}, \ldots, K_{m}^{0}\right\}$. We often repeat $\Omega_{0}$ 
in space $n$-times to generate the entire domain $\Omega=\bigcup_{i=1}^{n} \Omega_{i}$, along with the mesh $\mathcal{T}$ that discretizes the domain $\Omega, \mathcal{T}=\bigcup_{i=1}^{n} \mathcal{T}_{i}=\left\{K_{1}, K_{2}, \ldots, K_{m \cdot n}\right\}$, where $\mathcal{T}_{i}$ is the discretization of each $\Omega_{i}$.

We define the operator $\mathcal{F}: K_{j} \in \mathcal{T} \longmapsto K_{i}^{0} \in \mathcal{T}_{0}$ such that given an element $K_{j} \in \mathcal{T}$ it returns the corresponding element in the master mesh $\mathcal{T}_{0}$.

The matching of materials and geometry associated with multiple copies of the same original rock allows us to write for one element:

$$
\int_{K} \frac{k^{2}}{\rho} \phi_{j} \phi_{i}=\int_{\mathcal{F}(K)} \frac{k^{2}}{\rho} \phi_{j} \phi_{i} .
$$

To extend this equality to all terms of the bilinear form, we compute the submatrices of each element of the master grid before assembling the global matrix. Then, in order to assemble the matrix associated with $\mathcal{T}$, we search and copy each element matrix into it.

In our application, we employ two sets of precomputed matrices: one for the original rock, that contains the transmitter and its surrounding refinements, and another one for the repeated rock without transmitter and refinements (see Figure 3).

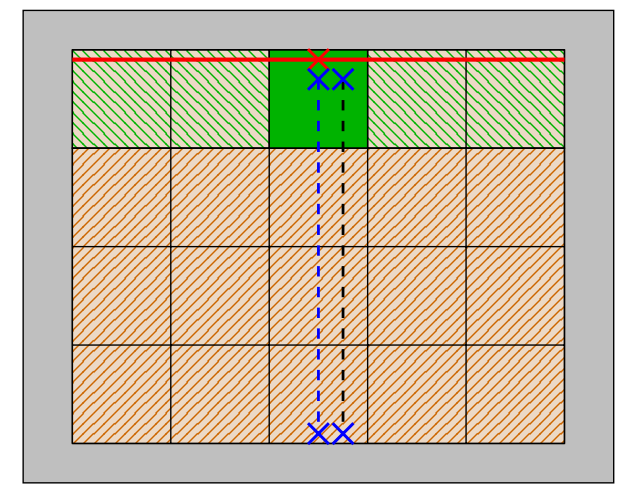

Figure 3: 2D model problem with the original rock in green repeated at one side in the propagation direction $(N=3)$ and at both sides in its perpendicular direction $(M=2)$. The transmitter, in red, covers the entire domain in the no-propagation direction. The red cross represents the center of the transmitter $T_{x}$. The blue and black dashed lines represent two lines of analysis. The blue crosses represent the first $\left(R_{x 1}\right)$ and last $\left(R_{x n}\right)$ receiver of each analysis line. We precompute two different matrices: one for the blocks of the green striped area that contains the transmitter, and another one for the blocks in the brown striped area. The PML zone is filled with gray color.

\subsubsection{From the FEM Solution to an Effective Velocity}

At low frequencies, a wave traveling through a heterogeneous media behaves as a sum of plane waves. Figure 4 shows the real part of the pressure computed by 
Equation (8). In such scenarios, Prony's method [45, 46] accurately estimates the effective velocity. From a uniform sampling of a signal, Prony's method builds a linear combination of damped complex exponentials to reconstruct the signal, providing valuable information such as amplitude, phase, damping, and phase velocity.



Figure 4: Real part of the solution at $1.27 \mathrm{kHz}$ in a 1D rock with a porous size equal to $125 \mathrm{~mm}$. The white background color indicates a material with $v_{\text {solid }}=4500 \mathrm{~m} / \mathrm{s}$; the gray background represents a material with $v_{\text {fluid }}=800$ $\mathrm{m} / \mathrm{s}$. The density of both materials is $1000 \mathrm{Kg} / \mathrm{m}^{3}$.

However, at high frequencies, the wavelength is comparable to the size of the pores, and the solution behaves as piecewise plane waves, one inside each sub-domain. Figure 5 s shows the real part of the pressure computed using (8). In this scenario, the plane wave assumption required by Prony's method is invalid, and we need to estimate the effective velocities differently. For that, we propose a simple method based on counting the number of wavelengths of the solution over the whole domain. To do so, we sample the solution $u_{h}$ as in the Prony's method, and we compute the phase difference of the pressure from one receiver to the next. In this way, we approximate the number of wavelengths located between one receiver and the next. By repeating the process throughout all receivers, we obtain an estimate of the total number of wavelengths $\left(\lambda_{t}\right)$ traveled by the wave. Then, we compute the velocity as:

$$
v_{e f f}=\frac{L \nu}{\lambda_{t}},
$$

where $\nu$ is the frequency in Hertz and $L$ is the distance between the first and last receiver. Experiment 1 in Section 4 compares Prony's method with the one based on counting the number of wavelengths. 




Figure 5: Real part of the solution at $48.3 \mathrm{kHz}$ in $1 \mathrm{D}$ rock with $125 \mathrm{~mm}$ as size of the pore. The white background color indicates a material with $v_{\text {solid }}=4500$ $\mathrm{m} / \mathrm{s}$; the gray background represents a material with $v_{\text {fluid }}=800 \mathrm{~m} / \mathrm{s}$. The density of both materials is $1000 \mathrm{Kg} / \mathrm{m}^{3}$.

\subsection{High Frequencies Analysis}

Approximation of high-frequency solutions by a FEM involves a prohibitive computational cost due to the need of considering several degrees of freedom per wavelength, which translates into large systems of equations. However, our application only requires to estimate the traveltime of the first arriving wave. To do so, we introduce the eikonal equation [48, which we solve with a FMM.

\subsubsection{Eikonal Equation}

We assume the solution of the wave equation (4) takes the following general form:

$$
p(t, \boldsymbol{x})=A(\boldsymbol{x}) e^{-i \omega(T(\boldsymbol{x})+t)},
$$

where $A$ is the amplitude, $\omega$ is the angular frequency and $T$ is a time function (the eikonal), which describes surfaces of constant phase (wavefronts) when $T$ is constant.

Plugging the ansatz (13) into the wave equation (4), separating real and imaginary parts and using the high-frequency assumption $(\omega \longrightarrow \infty)$ in the real part equation, we obtain the so called eikonal equation:

$$
|\nabla T(\boldsymbol{x})|^{2}=\frac{1}{c(\boldsymbol{x})^{2}} \text { in } \Omega
$$

where $T$ is the traveltime evaluated at each point $\boldsymbol{x}$ of the domain $\Omega$. 


\subsubsection{Fast Marching Method (FMM)}

A FMM is a finite difference scheme that solves boundary value problems with the unknown traveltime satisfying the eikonal equation:

$$
\left\{\begin{array}{l}
|\nabla T(\boldsymbol{x})|^{2}=\frac{1}{c(\boldsymbol{x})^{2}} \text { in } \Omega, \\
T(\boldsymbol{x})=f \text { at least on a part of } \partial \Omega,
\end{array}\right.
$$

where $f$ is typically 0 .

We consider a front given by the initial condition, which is systematically updated in the propagation direction (one grid point at a time), in a downwind fashion from known upwind values. This entropy-satisfying strategy preserves stability in the presence of a wavefront discontinuity, as explained in [57. The key ingredient of the method is to carefully select the order of traveltime evaluation based on the concept of narrow band: a set of points -that reproduce the shape of the wavefront- with pending traveltime evaluation. Since the wavefront propagates only forward, it crosses each point of the grid only once. The narrow band separates the points with known traveltimes from those with unknown values of $T$.

Traveltimes in the 3D narrow band for are updated by:

$$
\begin{array}{r}
{\left[\max \left(D_{i j k}^{-x} T,-D_{i j k}^{+x} T, 0\right)^{2}+\max \left(D_{i j k}^{-y} T,-D_{i j k}^{+y} T, 0\right)^{2}+\right.} \\
\left.+\max \left(D_{i j k}^{-z} T,-D_{i j k}^{+z} T, 0\right)^{2}\right]^{1 / 2}=\frac{1}{c_{i j k}},
\end{array}
$$

where $c_{i j k}$ is the velocity at grid point $(i, j, k)$ and $D_{i j k}$ are first order finite difference operators given by:

$$
\begin{aligned}
D_{i j k}^{-x} T=\frac{T_{i, j, k}-T_{i-1, j, k}}{\delta x}, & D_{i j k}^{+x} T=\frac{T_{i+1, j, k}-T_{i, j, k}}{\delta x} \\
D_{i j k}^{-y} T=\frac{T_{i, j, k}-T_{i, j-1, k}}{\delta y}, & D_{i j k}^{+y} T=\frac{T_{i, j+1, k}-T_{i, j, k}}{\delta y} \\
D_{i j k}^{-z} T=\frac{T_{i, j, k}-T_{i, j, k-1}}{\delta z}, & D_{i j k}^{+z} T=\frac{T_{i, j, k+1}-T_{i, j, k}}{\delta z},
\end{aligned}
$$

and $\delta_{x}, \delta_{y}$ and $\delta_{z}$ are the grid spacings in $x, y$, and $z$ directions, respectively. We select a grid conforming with the spatial resolution given by the tomography:

$$
\delta_{(\cdot)}=\frac{N_{(\cdot)}}{L_{(\cdot)}}
$$

where subscript (.) denote the spatial component, and $N_{(\cdot)}$ and $L_{(\cdot)}$ denote the number of voxels obtained in the tomography, and the dimension of the rock, respectively.

To initialize the narrow band, we compute analytically the traveltime in one end of the rock as:

$$
T_{i, j, k}=\frac{c_{i, j, k}}{2 \delta_{z}} .
$$


for $i \in 1,2, \ldots, N_{x}, j \in 1,2, \ldots, N_{y}$ and $k=1$.

The FMM algorithm reads:

1. Generate the grid conforming to the voxels.

2. Initialize the narrow band using Equation (19).

3. Do until all determining all traveltimes:

(a) Find the point with minimum traveltime in the narrow band.

(b) Update the narrow band.

(c) Update traveltimes at the points recently added to the narrow band and their neighbours using equation (16).

(d) Compute traveltimes on the bottom part of the domain using Equation 190 with $k=N_{z}$.

Figure 6 illustrates the sequence $3(\mathrm{a})-3(\mathrm{~b})$ in $2 \mathrm{D}$. The first order scheme described above is unconditionally stable [56]. For more details on FMM, we refer the reader to [52].



(a) Original narrow band

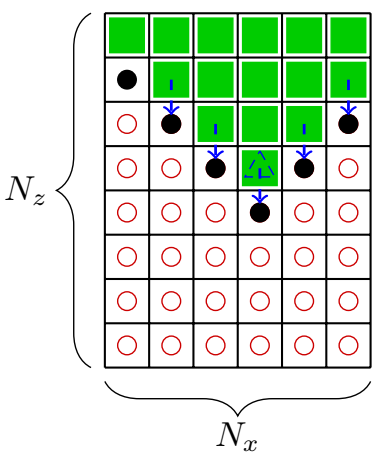

(b) Updated narrow band

Figure 6: Example of a narrow band evolution in a two dimensional domain: green squares stand for points where the traveltime is known; red empty circles are points with unknown travel time; black filled circles are the points in the narrow band. (a) A blue triangle identifies the minimum traveltime in the narrow band. (b) The minimum and its neighbors of the narrowband (until correcting all arising discontinuities) are tagged as known traveltime points, let advancing the narrow band.

In $1 \mathrm{D}$, a FMM reduces to computing the traveltime from one point of the grid to the next one, using the spacing between the two points and the known velocity at the arrival point. 


\section{Numerical Results}

This section contains multiple numerical results. Experiments 1-4 consider 1D periodic and non-periodic formations, and assess the performance of the methods for different values of the involved parameters (e.g., number of rock repetitions and number of wavelengths needed in the domain). Experiments 5-8 show the influence of the principal physical quantities (e.g., density, porosity, the size of a pore, and different pore distribution) in the effective velocity. Experiment 9 describes the effective velocity profile computed for four nonperiodic formations. Experiment 10 analyzes the lateral extension of the domain for a 2D rock. Experiment 11 exhibits a 2D VTI rock. It also shows the match between the Time-Average formula and FMM in the high-frequency regime - Experiment 12 employs HTI rocks and shows the discrepancy between the Time-Average formula and the FMM. Then, experiment 13 further validates the FMM. Finally, experiment 14 applies the proposed methods to two real rocks.

\subsection{Samples and Modeling Considerations}

We consider samples composed of two materials. We identify solid (S) and fluid (F) pixels with white and black color, respectively (see Table 1).

Table 1: Nomenclature associated to each one-dimensional pixel.

\begin{tabular}{llll}
\hline $\begin{array}{l}\text { Graphical } \\
\text { representation }\end{array}$ & $\begin{array}{l}\text { Material } \\
\text { state }\end{array}$ & $\begin{array}{l}\text { Material } \\
\text { properties }\end{array}$ \\
\hline$\square$ & Solid (S) & $v_{\text {solid }}$ & $\rho_{\text {solid }}$ \\
$\square$ & Fluid (F) & $v_{\text {fluid }}$ & $\rho_{\text {fluid }}$ \\
\hline
\end{tabular}

We generate particular periodic samples by repeating a specific sequence of pixels several times. To refer to a periodical 1D synthetic rock, we introduce the following nomenclature: we first indicate that it is a one-dimensional (1D) periodic $(\mathrm{P})$ rock with the abbreviation 1DP. Next, we write the number of total pixels that compose the sample and finally, inside brackets, the repeated sequence of pixels. Table 2 shows two periodic samples.

Table 2: Nomenclature and porosity associated with two one-dimensional periodic formations.

\begin{tabular}{|c|c|c|}
\hline Graphical representation & Nomenclature & Porosity \\
\hline & 1DP8(7S1F) & $12.5 \%$ \\
\hline & 1DP8(3S1F) & $25 \%$ \\
\hline
\end{tabular}


We additionally consider four large realistic non-periodic formations composed each of 1203 pixels. Table 3 summarizes the nomenclature and the porosity of each non-periodic formation.

Table 3: Nomenclature and porosity of non-periodic one-dimensional formations.

\begin{tabular}{ll||ll}
\hline Nomenclature & Porosity & Nomenclature & Porosity \\
\hline 1D Rock 1 & $11.31 \%$ & 1D Rock 3 & $21.36 \%$ \\
1D Rock 2 & $22.61 \%$ & 1D Rock 4 & $25.85 \%$ \\
\hline
\end{tabular}

Figure 7 describes the one-dimensional model of the formation and identifies the necessary geometric model parameters.



Figure 7: 1D Model problem. The transmitter (in red) is defined by its central point $\mathrm{T}_{x}$ and size $\mathrm{T}_{s}$. The blue crosses with coordinates $\mathrm{R}_{x 1}$ and $\mathrm{R}_{x n}$ indicate the first and last receiver, respectively.

We denote the domain of the sample by $\Omega_{0}=(a, b)$. To truncate the computational domain, we introduce a PML on the outer part of the domain. We automatically select its size $\left|a-a^{\prime}\right|=\left|b-b^{\prime}\right|$ as the minimum between $10 \%$ of the domain of the sample and three times the size of the largest wavelength, i.e.:

$$
\left|a-a^{\prime}\right|=\left|b-b^{\prime}\right|=\min \left(0.1(b-a), 3 \lambda_{\text {fast }}\right),
$$

where $\lambda_{\text {fast }}$ is the wavelength associated with the fastest material of the rock (in our case, the solid, i.e., $\lambda_{\text {fast }}=\lambda_{\text {solid }}$ ).

We often extend the analysis domain by repeating the sample. We can repeat the original rock only at one side (see Figure 8a), or at both sides (see Figure 8b).

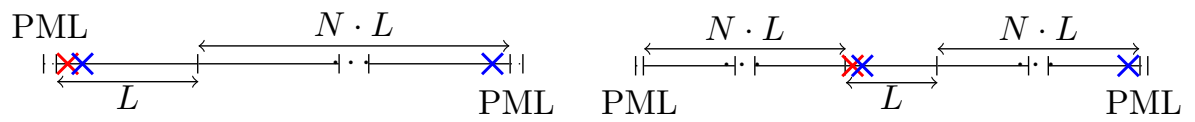

(a) Rock repeated $N$ times at one side of the(b) Rock repeated $N$ times at both sides of the transmitter transmitter

Figure 8: 1D model problem. The red cross represents the transmitter and the blue crosses the first and last receiver. 
We consider a domain $\Omega_{0}=(-0.5,0.5)^{d}$ meters, where $d$ is the spatial dimension. A rescaling to a different sample size is straightforward. We place the transmitter $0.05 \mathrm{~m}$ away from the closest boundary, and the receivers at least 0.10 away from any boundary, following a straight line. In the Finite Element experiments, we select the polynomial order of approximation $p=2$.

Table 4: Nomenclature and porosity associated with 2D periodic VTI and HTI formations.

\begin{tabular}{lll}
\hline $\begin{array}{l}\text { Graphical } \\
\text { representation }\end{array}$ Nomenclature & Porosity \\
\hline
\end{tabular}



We also generate a synthetic formation of $1200 \times 1200$ pixels that we denote as the labyrinth (see Figure 9). In this formation, the fastest wave travels through the solid, circumventing the black fluid material.

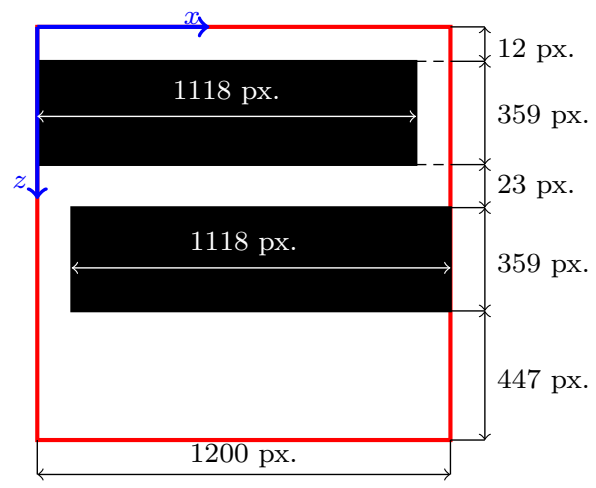

Figure 9: Sketch of a synthetic formation denoted as the labyrinth composed by $1200 \times 1200$ pixels. The black region represents the fluid material with P-wave velocity slower than the solid material identified with white color.

Table 5 shows three types of 3D layered periodic formations. In the first 
formation with VTI symmetry, the normal vector of each layer points towards the direction of the main $\mathrm{P}$-wave propagation direction (z-axis). The other two formations with HTI symmetry have the normal vector of each layer pointing perpendicularly to the main $\mathrm{P}$-wave propagation direction. The subscript $(x$ or $y$ ) denotes the normal vector of the layers. Finally, we consider two

Table 5: Nomenclature and porosity associated with 3D periodic formations.

\begin{tabular}{lll}
\hline $\begin{array}{l}\text { Graphical } \\
\text { representation }\end{array}$ & Nomenclature & Porosity \\
\hline
\end{tabular}



3DVTI $12 \times 12 \times 12(1 \mathrm{~F} 2 \mathrm{~S}) \quad 33.3 \%$



$3 \mathrm{DHTI}_{x} 12 \times 12 \times 12(1 \mathrm{~F} 3 \mathrm{~S}) \quad 25 \%$



$3 \mathrm{DHTI}_{y} 12 \times 12 \times 12(1 \mathrm{~F} 3 \mathrm{~S}) \quad 25 \%$

heterogeneous real rock samples we analyzed in the laboratory with a microCT scan. The main $\mathrm{P}$-wave propagation direction is along the last dimension ( $z$-axis). The postprocessed data extracted from the micro-CT is a set of 1618 images of $1200 \times 1200$ pixels each. The domain is $\Omega_{0}=1.547 \times 1.547 \times 2.086 \mathrm{~cm}$. Each pixel of the micro-CT map corresponds to a square of 12.89 micrometers. To reduce the computational cost, we sample over one vertical cross-section of $1200 \times 1618$ pixels extracted from the samples.

(a) The first rock, denoted as 3D Rock 1, is composed of five materials. Table 6 summarizes the number assigned to each material, its volume fraction percentage, and properties.

(b) The second rock, denoted as 3D Rock 2, is composed of three materials. 
Table 6: Material number, volume fraction, wave velocity and density of the 3D Rock 1 constituents.

\begin{tabular}{llll}
\hline $\begin{array}{l}\text { Material } \\
\text { number }(i)\end{array}$ & $\begin{array}{l}\text { Volume } \\
\text { fraction }\left(\phi_{i}\right)\end{array}$ & $c_{i}(\mathrm{~m} / \mathrm{s})$ & $\rho_{i}\left(\mathrm{~kg} / \mathrm{m}^{3}\right)$ \\
\hline 1 & 0.800185 & 6041.98 & 2648.0 \\
2 & 0.034559 & 2424.87 & 1373.0 \\
3 & 0.071186 & 1513.27 & 1000.0 \\
4 & 0.046283 & 2138.54 & 1298.4 \\
5 & 0.047787 & 1493.02 & 1149.2 \\
\hline
\end{tabular}

Table 7 summarizes the number assigned to each material, its volume fraction percentage, and properties.

Table 7: Material number, volume fraction, wave velocity and density of the 3D Rock 3 constituents.

\begin{tabular}{llll}
\hline $\begin{array}{l}\text { Material } \\
\text { number }(i)\end{array}$ & $\begin{array}{l}\text { Volume } \\
\text { fraction }\left(\phi_{i}\right)\end{array}$ & $c_{i}(\mathrm{~m} / \mathrm{s})$ & $\rho_{i}\left(\mathrm{~kg} / \mathrm{m}^{3}\right)$ \\
\hline 1 & 0.619703 & 6645.11 & 2710.0 \\
2 & 0.354040 & 5161.60 & 2197.0 \\
3 & 0.026257 & 1513.27 & 1000.0 \\
\hline
\end{tabular}

Table 8 summarizes the simulation parameters used in each experiment.

\subsection{Experiment 1: Prony vs Count Wavelength}

In this experiment, we extend the domain at both sides of the original sample to guarantee $10 \lambda_{\text {solid }}$ at each side.

Figure 10 shows the theoretical effective velocities computed by equations (2) and (3), and compares the profiles of compressional wave effective velocities obtained by the application of Prony's method (red line) and counting the number of wavelengths (green line) for two periodic rocks. We observe that Prony's method converges only in the low-frequency regime, which is the region where the solution is given by a sum of plane waves. The count wavelengths method properly estimates velocities at low frequencies, matching the results obtained by Prony's method. It also performs adequately in the high-frequency regime and converges to the theoretical solution $v_{\text {eff }}^{T A}$ given by the Wyllie timeaverage. 
Table 8: Summary of simulation parameters used in each experiment.

\begin{tabular}{|c|c|c|c|c|c|c|c|c|c|}
\hline $\begin{array}{l}\text { Experiment } \\
\text { number }\end{array}$ & $\begin{array}{l}\text { Figure } \\
\text { table n }\end{array}$ & or & $\begin{array}{l}\text { Formation } \\
\text { nomenclature }\end{array}$ & $\begin{array}{l}c_{\text {solid }} \\
(\mathrm{m} / \mathrm{s})\end{array}$ & $\begin{array}{l}\rho_{\text {solid }} \\
\left(\mathrm{kg} / \mathrm{m}^{3}\right)\end{array}$ & $\begin{array}{l}c_{\text {fluid }} \\
(\mathrm{m} / \mathrm{s})\end{array}$ & $\begin{array}{l}\rho_{\text {fluid }} \\
\left(\mathrm{kg} / \mathrm{m}^{3}\right)\end{array}$ & $\begin{array}{l}\text { Precomputed } \\
\text { matrix }\end{array}$ & $\begin{array}{l}\text { Fitting } \\
\text { meshes }\end{array}$ \\
\hline \multirow{2}{*}{1} & Figure & $10 \mathrm{a}$ & 1DP8(3S1F) & \multirow{2}{*}{4500} & \multirow{2}{*}{2800} & \multirow{2}{*}{800} & \multirow{2}{*}{1000} & \multirow{2}{*}{ No } & \multirow{2}{*}{ No } \\
\hline & Figure & $10 \mathrm{~b}$ & 1DP8(7S1F) & & & & & & \\
\hline \multirow{2}{*}{2} & Table & & 1D Rock 1-4 & \multirow{2}{*}{4500} & 1000 & \multirow{2}{*}{800} & \multirow{2}{*}{1000} & $*$ & $*$ \\
\hline & Figure & 11 & 1D Rock 4 & & $*$ & & & Yes & Yes \\
\hline \multirow{2}{*}{3} & Figure & $12 \mathrm{a}$ & 1DP8(3S1F) & \multirow{2}{*}{4500} & \multirow{2}{*}{2800} & \multirow{2}{*}{800} & \multirow{2}{*}{1000} & \multirow{2}{*}{ No } & \multirow{2}{*}{ Yes } \\
\hline & Figure & $12 \mathrm{~b}$ & 1DP8(7S1F) & & & & & & \\
\hline \multirow{2}{*}{4} & Figure & $13 a$ & 1D Rock 1 & \multirow{2}{*}{4500} & \multirow{2}{*}{1000} & \multirow{2}{*}{800} & \multirow{2}{*}{1000} & \multirow{2}{*}{ Yes } & \multirow{2}{*}{ Yes } \\
\hline & Figure & $13 \mathrm{~b}$ & 1D Rock 2 & & & & & & \\
\hline \multirow{2}{*}{5} & Figure & $14 a$ & 1DP8(3S1F) & \multirow{2}{*}{4500} & \multirow{2}{*}{$*$} & \multirow{2}{*}{800} & $*$ & Yes & Yes \\
\hline & Figure & $14 \mathrm{~b}$ & 1DP8(7S1F) & & & & & & \\
\hline 6 & Figure & $15 \mathrm{a}$ & 1DP8(1S1F) & 4500 & 2800 & 800 & 1000 & Yes & Yes \\
\hline & Figure & $15 \mathrm{~b}$ & $\begin{array}{l}\text { 1DP8(3S1F) } \\
\text { 1DP8(7S1F) }\end{array}$ & & 1000 & & & & \\
\hline & Figure & $16 a$ & 1DP8(1S1F) & & 2800 & & & & \\
\hline 7 & Figure & $16 \mathrm{~b}$ & $\begin{array}{l}\text { 1DP80(1S1F) } \\
1 \mathrm{DP} 800(1 \mathrm{~S} 1 \mathrm{~F})\end{array}$ & 4500 & 1000 & 800 & 1000 & Yes & Yes \\
\hline & Figure & $17 \mathrm{a}$ & $\begin{array}{l}\text { 1DP8(3S1F }) \\
\text { 1DP80(3S1F }) \\
\text { 1DP800(3S1F })\end{array}$ & & 2800 & & & & \\
\hline & Figure & $17 \mathrm{~b}$ & $\begin{array}{l}\text { 1DP8(7S1F) } \\
\text { 1DP80(7S1F) } \\
\text { 1DP800(7S1F })\end{array}$ & & & & & & \\
\hline 8 & Figure & $18 \mathrm{a}$ & $*$ & 4500 & 2800 & 800 & 1000 & Yes & Yes \\
\hline & Figure & $18 \mathrm{~b}$ & & 1000 & 2000 & 0 & 1000 & & $10 \mathrm{~s}$ \\
\hline 9 & Figure & 19 & 1D Rock 1-4 & 4500 & 2800 & 800 & 1000 & Yes & Yes \\
\hline 10 & Figure & 20 & 2DVTI 12x12 (1F2S) & 4500 & 1000 & 800 & 1000 & Yes & Yes \\
\hline & Figure & $22 \mathrm{a}$ & 1DP12(1S1F) & 4500 & 2800 & 800 & 1000 & Yes & Yes \\
\hline 11 & Figure & $22 \mathrm{~b}$ & 2DVTI (1F2S) & & 1000 & & & & \\
\hline & Table & 11 & $\begin{array}{l}\text { 2D VTI (1F2S) } \\
\text { 2D VTI (1F3S) } \\
\text { 2D VTI (1F7S) } \\
\text { 3D VTI (1F3S) }\end{array}$ & & - & & - & - & - \\
\hline & Figure & $23 \mathrm{a}$ & 2DHTI (1F2S) & 4500 & 2800 & 800 & 1000 & Yes & Yes \\
\hline 12 & Figure & $23 \mathrm{~b}$ & & & 1000 & & & & \\
\hline & Table & 12 & $\begin{array}{l}\text { 2D HTI }(1 \mathrm{~F} 2 \mathrm{~S}) \\
\text { 2D HTI }(1 \mathrm{~F} 3 \mathrm{~S}) \\
\text { 2D HTI }(1 \mathrm{~F} 7 \mathrm{~S}) \\
\text { 3D } \mathrm{HTI}_{x}(1 \mathrm{~F} 3 \mathrm{~S}) \\
\text { 3D } \mathrm{HTI}_{y}(1 \mathrm{~F} 3 \mathrm{~S})\end{array}$ & & - & & - & - & - \\
\hline 13 & Figure & $24 \mathrm{~b}$ & Labyrinth & 4500 & - & 800 & 1000 & - & - \\
\hline 14 & Figure & 25 & 3D Rock 1 & $*$ & $*$ & $*$ & $*$ & Yes & Yes \\
\hline & Figure & 26 & 3D Rock 2 & & & & & & \\
\hline
\end{tabular}

Symbol * indicates that the corresponding field is a variable explained in its own experiment subsection.

Symbol - indicates that such field is not applicable in the considered experiment. 




(a) Rock 1DP8(3S1F)

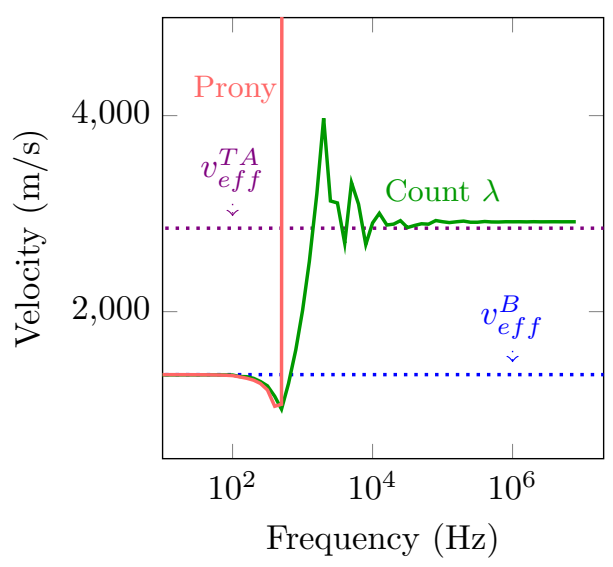

(b) Rock 1DP8(7S1F)

Figure 10: Effective compressional wave velocities computed with Prony's method (red line) and counting wavelengths (green line).

\subsection{Experiment 2: Precomputed-Matrix and Fitting vs Non-Fitting Meshes}

In this experiment, we assess the accuracy of non-fitting meshes and precomputed matrix technique. We compute the compressional wave effective velocity using Prony's method for the non-periodic formations displayed in Table 3 , excited at a frequency of $10 \mathrm{~Hz}$. We extend the domain at both sides of the original sample virtually to guarantee $1 \lambda_{\text {Backus }}$ at each side.

Table 9 shows the effective velocity (in $\mathrm{m} / \mathrm{s}$ ) computed over the non-periodic formations defined in Table 3 using the three different methods listed below.

- Method 1 (M1) employs a FEM with a traditional fitting mesh technique.

- Method 2 (M2) uses a FEM with a non-fitting mesh with only one element in each repeated rock and without a precomputed matrix.

- Method 3 (M3) considers a FEM with a non-fitting mesh and a precomputed matrix.

This table also exhibits the Backus effective velocity $v_{\text {eff }}^{B}$ given by equation [2].

Table 9 reveals that the computation of the effective velocity through any of the aforementioned methods provides an excellent approximation to the Backus average velocity $v_{\text {eff }}^{B}$ (see equation 22. Moreover, the results obtained with methods two and three coincide. This validates the implementation of the precomputed matrix technique. The solution associated with a non-fitting mesh differs slightly from the one computed with a fitting mesh. To assess these discrepances, Figure 11 shows the velocity computed with non-fitting meshes 
Table 9: Comparison between the theoretical compressional wave effective velocity $v_{\text {eff }}^{B}$ and the computed one (using Prony) from the FEM solution over the non-periodic formations using three different methods: M1 employs fitting meshes; M2 uses a non-fitting mesh without precomputed matrix; and M3 considers a non-fitting mesh with precomputed matrix. The values for the velocity are in $\mathrm{m} / \mathrm{s}$.

\begin{tabular}{lllll}
\hline Formation & $v_{\text {eff }}^{B}$ & M1 & M2 & M3 \\
\hline 1D Rock 1 & 2129.870 & 2129.866 & 2129.870 & 2129.870 \\
1D Rock 2 & 1598.210 & 1598.222 & 1598.220 & 1598.220 \\
1D Rock 3 & 1638.169 & 1638.205 & 1638.199 & 1638.199 \\
1D Rock 4 & 1506.608 & 1506.513 & 1506.547 & 1506.547 \\
\hline
\end{tabular}

against the number of subcells for different values of the fast material density, in the case of $1 \mathrm{D}$ Rock 4 . For a large number of subcells per element, the error increases as function of the ratio between densities.

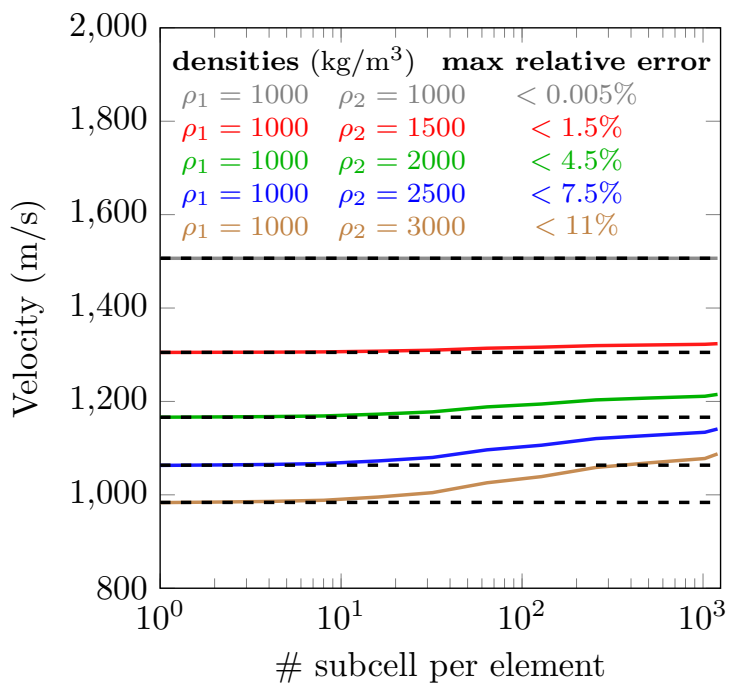

Figure 11: Influence of the number of subcells per element in the estimated effective compressional wave velocity solution. Non-periodic 1D Rock 4 sample excited at $10 \mathrm{~Hz}$ for multiple densities.

The optimal number of macro-elements to have the numerical error under control depends upon multiple parameters, including: (a) the user-selected tolerance error, (b) the frequency, (c) the ratio between densities, (d) the number of subcells in the original rock, and (e) the size of the original domain. For the cases showed in Figure 11, we limit ourselves to $\left\lfloor\left(\rho_{2} / \rho_{1}\right)^{\rho_{2} / \rho_{1}}\right\rfloor$ macro-elements to discretize the original rock. Moreover, we consider a similar number of subcells 
for each macro-element.

\subsection{Experiment 3: Rock-repetitions: One Side vs Two Sides}

We consider two periodic rocks. Figure 12 shows the effective velocity (obtained counting wavelengths) in the original rock, as well as the effect of repeating the rock at one or both sides of the original rock.

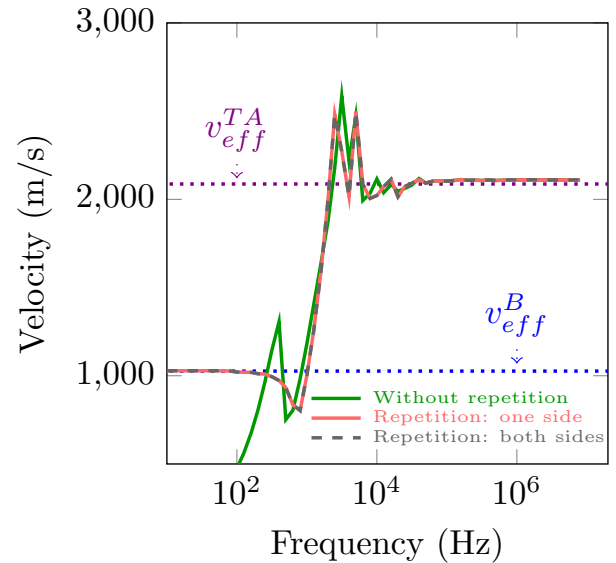

(a) Rock 1DP8(3S1F)



(b) Rock 1DP8(7S1F)

Figure 12: Effective compressional wave velocities computed counting wavelengths on different domain sizes.

At low frequencies, the effective velocity is different from the Backus velocity $v_{e f f}^{B}$ when considering only the original rock. But if we repeat it at one or both sides, then the two velocities match. A one-side repetition is computationally more efficient. At high frequencies, all simulations converge to $v_{e f f}^{T A}$.

The results of this experiment indicate we should repeat the rock at one side and guarantee a minimum number of wavelengths in the domain.

\subsection{Experiment 4: Rock-repetitions: Number of Wave- lengths}

This experiment analyzes the number of times we should repeat the rock. For that, we use the samples 1D Rock 1 and 1D Rock 2.

Figure 13 studies the effect of the number of wavelengths contained in the domain on the effective velocity. We consider three cases: 10, 3, and 1 wavelengths of the solid. Aside from slight differences in the solution, all of them provide good approximations of the quantity of interest. 


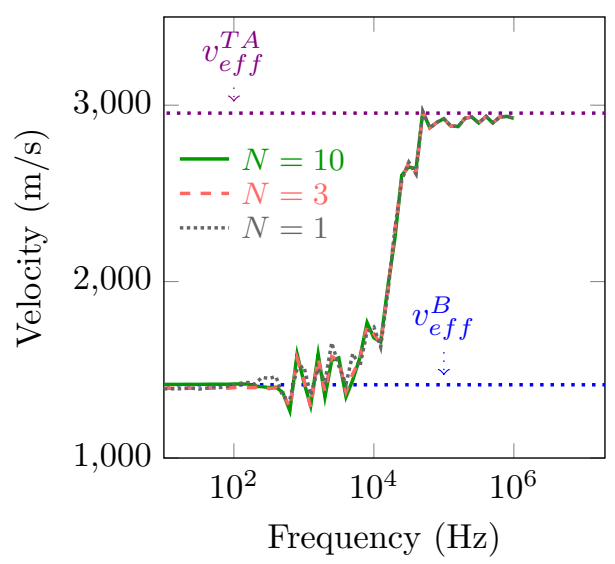

(a) 1D Rock 1

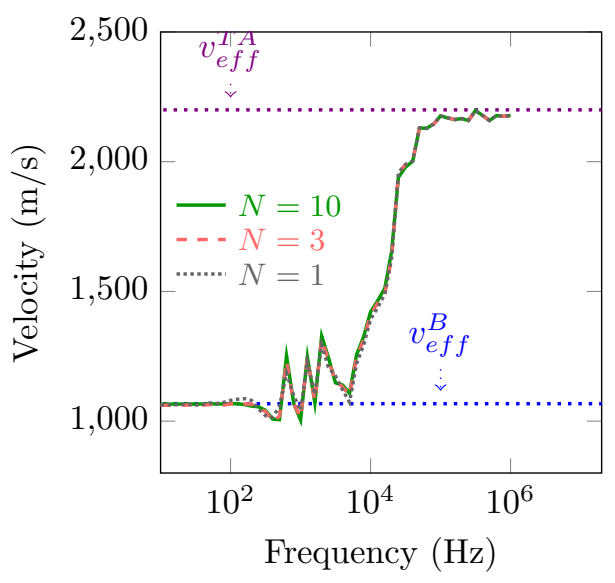

(b) $1 \mathrm{D}$ Rock 2

Figure 13: Effective compressional wave velocities computed counting wavelengths. The repetition of the rock at only one side guarantees a domain size of $N \lambda_{\text {solid }}$.

\subsection{Experiment 5: Influence of Density}

At low frequencies, an increase in the density of the fast material diminishes the effective velocities (see Figure 11). Figure 14 investigates the effect of different density ratios $\rho_{\text {solid }} / \rho_{\text {fluid }}$ on the compressional wave velocity for two periodic rocks. The one-side repetition of the rock guarantees a domain size of $1 \lambda_{\text {solid }}$.

We observe a decrease of the effective compressional wave velocity with the increase of the ratio $\rho_{\text {solid }} / \rho_{\text {fluid }}$ at low frequencies. The frequency that separates the Backus average zone and the transition zone takes smaller values when increasing this ratio. At high frequencies, the value of the compressional wave velocity is independent of the densities. These two observations agree with the theoretical limits discussed in Section 2

\subsection{Experiment 6: Influence of Porosity}

We consider the samples 1DP8(1S1F), 1DP8 $(3 \mathrm{~S} 1 \mathrm{~F})$ and $1 \mathrm{DP} 8(7 \mathrm{~S} 1 \mathrm{~F})$ with porosities equal to $50 \%, 25 \%$, and $12.5 \%$, respectively. We repeat the rock at one-side to guarantee a domain size of $1 \lambda_{\text {solid }}$. Figure 15 shows a decrease on the effective compressional wave velocity when the porosity diminishes. The three curves display a qualitatively similar profile -in terms of oscillations and frequency limits- in the transition zone. The frequency that separates the Backus average zone from the transition zone increases when augmenting the porosity. 


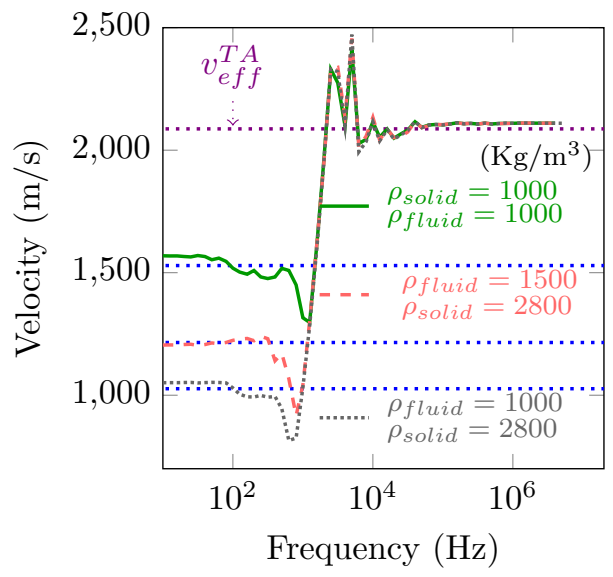

(a) Rock 1DP8(3S1F)

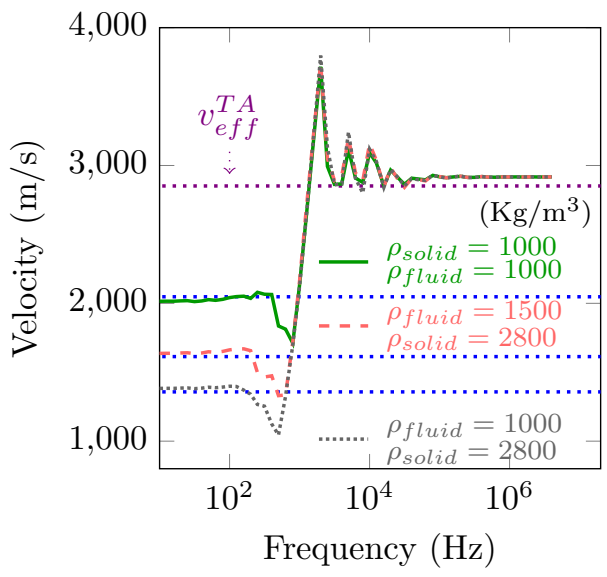

(b) Rock 1DP8 $(7 \mathrm{~S} 1 \mathrm{~F})$

Figure 14: Effective compressional wave velocities computed counting wavelengths with different density ratios.

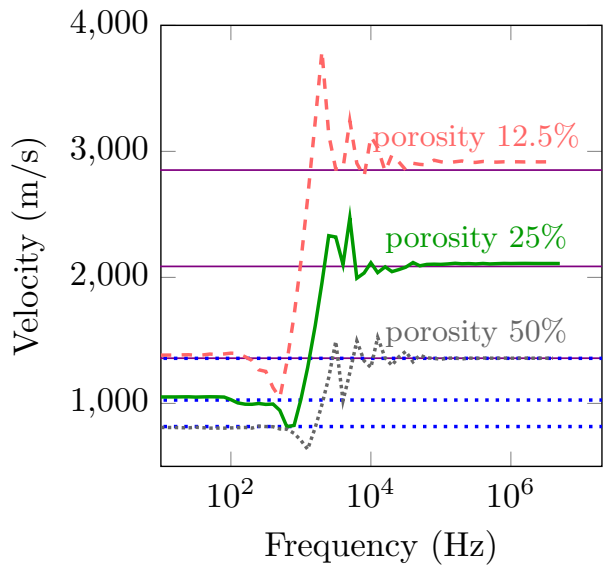

(a) $\rho_{\text {solid }}=2800 \mathrm{Kg} / \mathrm{m}^{3}$

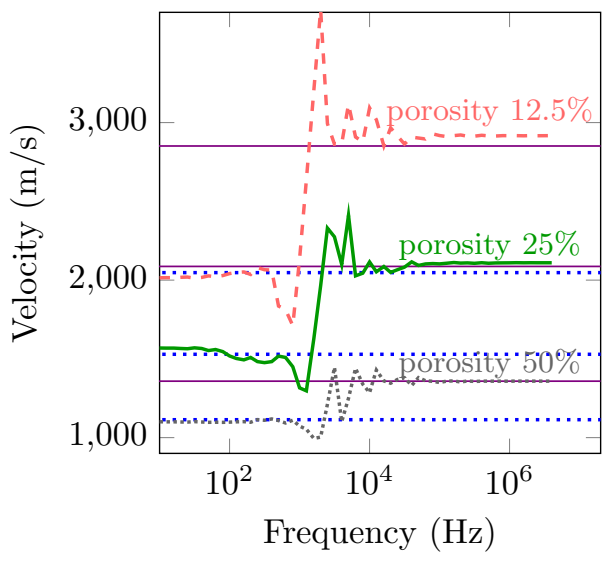

(b) $\rho_{\text {solid }}=1000 \mathrm{Kg} / \mathrm{m}^{3}$

Figure 15: Effective compressional wave velocities computed counting wavelengths in the rock $1 \mathrm{DP} 8(3 \mathrm{~S} 1 \mathrm{~F})$-green line-, $1 \mathrm{DP} 8(7 \mathrm{~S} 1 \mathrm{~F})$-red line- and 1DP8(1S1F) -dotted gray line-- 


\subsection{Experiment 7: Influence of the Size of the Pore}

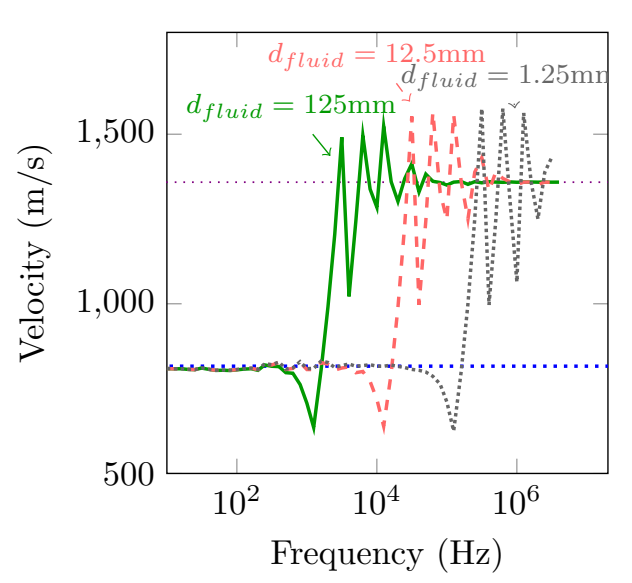

(a) $\rho_{\text {solid }}=2800 \mathrm{Kg} / \mathrm{m}^{3}$

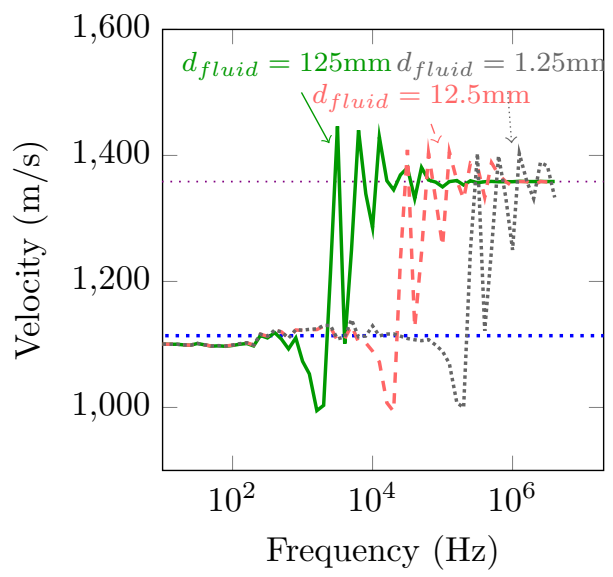

(b) $\rho_{\text {solid }}=1000 \mathrm{Kg} / \mathrm{m}^{3}$

Figure 16: Effective compressional wave velocities computed counting wavelengths in samples with different sizes of the pore $\left(d_{\text {fluid }}\right)$.

In this experiment, we repeat the rock on one-side to guarantee $1 \lambda_{\text {solid }}$. Figure 16 illustrates the behavior of the effective velocity in three synthetic rocks: $1 \mathrm{DP} 8(1 \mathrm{~S} 1 \mathrm{~F}), 1 \mathrm{DP} 8(1 \mathrm{~S} 1 \mathrm{~F})$ and $1 \mathrm{DP} 8(1 \mathrm{~S} 1 \mathrm{~F})$, all of them of porosity $50 \%$ and size of the pores $\left(d_{\text {fluid }}\right)$ equal to $125,12.5$, and 1.25 millimeters, respectively.

Figure 17a considers the synthetic rocks 1DP8(3S1F), 1DP80(3S1F) and 1DP800(3S1F) with porosity $25 \%$. Figure $17 \mathrm{~b}$ employs the rocks 1DP8(7S1F), $1 \mathrm{DP} 80(7 \mathrm{~S} 1 \mathrm{~F})$ and $1 \mathrm{DP} 800(7 \mathrm{~S} 1 \mathrm{~F})$ with porosity $12.5 \%$.

In all case of Figures 16 and 17 we observe a shift of the curves on frequency as the size of the pore decreases. We conclude the shift is independent of the porosity and density. These results agree with the theoretical 1D results (see Figure 1): the Backus average zone appears when the ratio between the wavelength and the size of the pore is greater than one. When this ratio is much smaller than one, we are in the time average zone.

\subsection{Experiment 8: Influence of the Distribution of the Sizes of the Pore}

We consider rocks with a specific size of the pore distribution by joining the rocks $1 \mathrm{DP} 400(150 \mathrm{~T} 50 \mathrm{~F})$ with $1 \mathrm{DP} 400(3 \mathrm{~T} 1 \mathrm{~F})$-porosity equal to $25 \%-$, and 1DP400(350T50F) with 1DP400(7T1F) - porosity equal to $12.5 \%$-.

Figure 18 illustrates how the transition zone between low and high frequencies is wide if there are pores with different sizes in the same rock. In this case, the frequency that indicates the change from the Backus average zone 


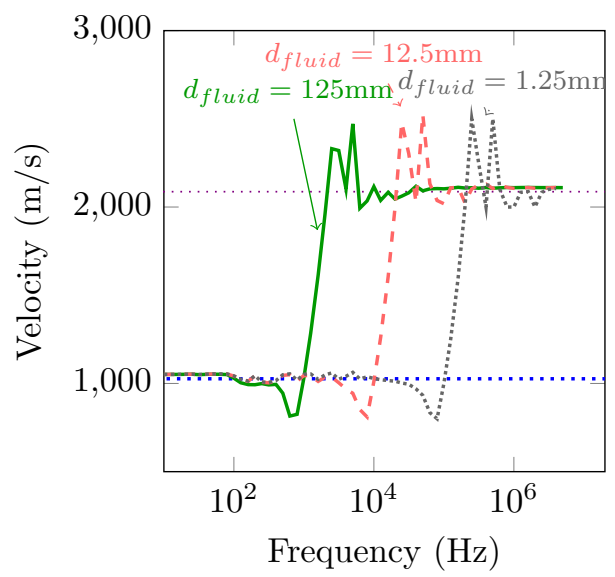

(a) porosity of $25 \%$

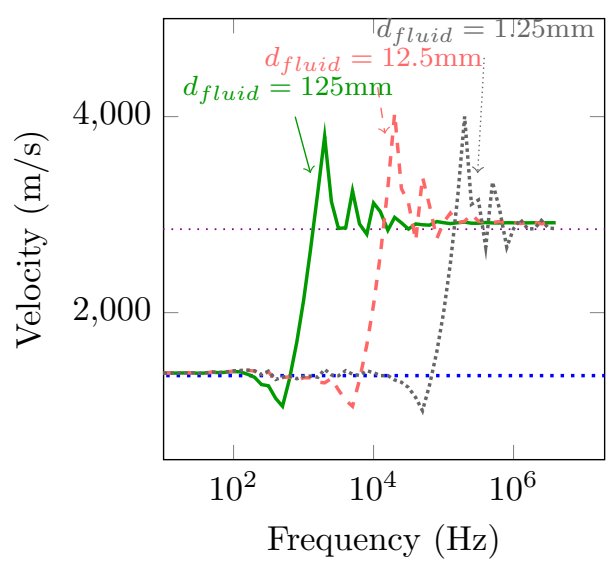

(b) porosity of $12.5 \%$

Figure 17: Effective compressional wave velocities computed counting wavelengths in samples with different sizes of the pore $\left(d_{\text {fluid }}\right)$.

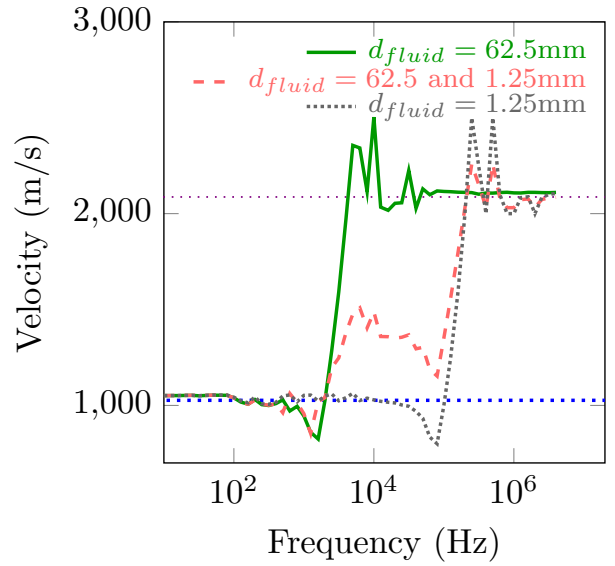

(a) porosity of $25 \%$

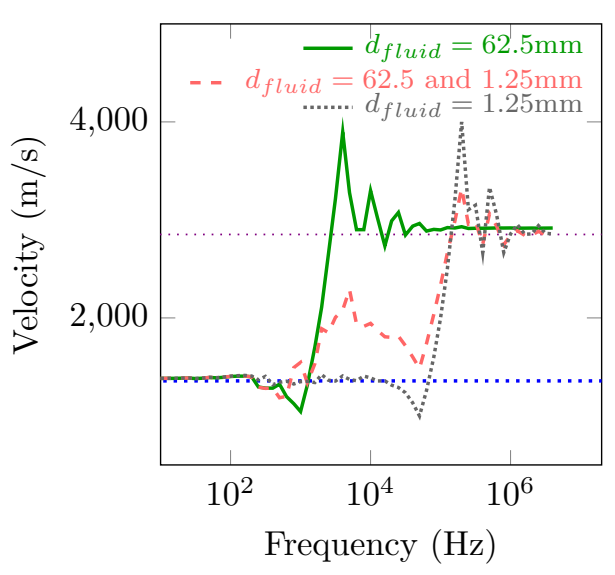

(b) porosity of $12.5 \%$

Figure 18: Effective compressional wave velocities computed counting wavelengths in samples with different size of the pore $\left(d_{\text {fluid }}\right)$.

to the transition zone is related to the largest size of the pore, whereas the frequency from the transition zone to the time average zone is associated with the smallest size of the pore. 


\subsection{Experiment 9: 1D Non-periodical Formations}

Figure 19 displays the effective velocity profiles over the rocks displayed in Table 3. The profiles converge to the theoretical bounds at low and high frequencies.

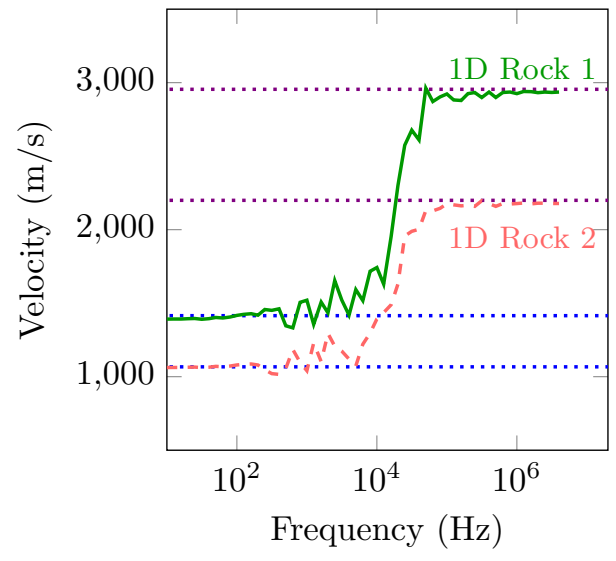

(a) 1D Rock 1 and 2

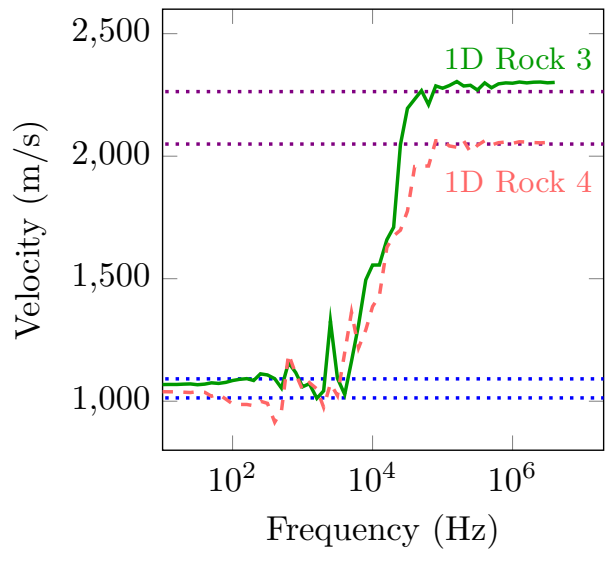

(b) $1 \mathrm{D}$ Rock 3 and 4

Figure 19: Effective compressional wave velocities computed counting wavelengths for the non-periodic rocks.

To assess FMM's efficiency, we compare its effective velocities with the Wyllie time average $v_{\text {eff }}^{T A}$. Table 10 shows that the two methods deliver equivalent results in $1 \mathrm{D}$.

Table 10: Effective velocities obtained for non-periodical rocks in the highfrequency regime, with Time-Average and FMM

\begin{tabular}{llll}
\hline Formation & Porosity & $v_{\text {eff }}^{T A}(\mathrm{~m} / \mathrm{s})$ & FMM $(\mathrm{m} / \mathrm{s})$ \\
\hline 1D Rock 1 & $11.31 \%$ & 2954.9672 & 2954.9673 \\
1D Rock 2 & $22.61 \%$ & 2199.7156 & 2199.7156 \\
1D Rock 3 & $21.36 \%$ & 2263.5234 & 2265.7145 \\
1D Rock 4 & $25.85 \%$ & 2049.5007 & 2051.2966 \\
\hline
\end{tabular}

\subsection{Experiment 10: Lateral Extension}

This experiment analyzes the importance of the domain size in the perpendicular direction to the main $\mathrm{P}$-wave propagation direction (controlled by the parameter $M)$. For this, we test the sample $2 \mathrm{DVTI} 12 \mathrm{x} 12(1 \mathrm{~F} 2 \mathrm{~S})$. We postprocess the solution over one vertical line located at the center of the rock. 
Figure 20 reveals that at low frequencies, the domain should be repeated along its lateral dimension (perpendicular direction to the main $\mathrm{P}$-wave propagation direction). In the remaining experiments, we will select $\mathrm{M}$ to guarantee at least one $\lambda_{\text {solid }}$.

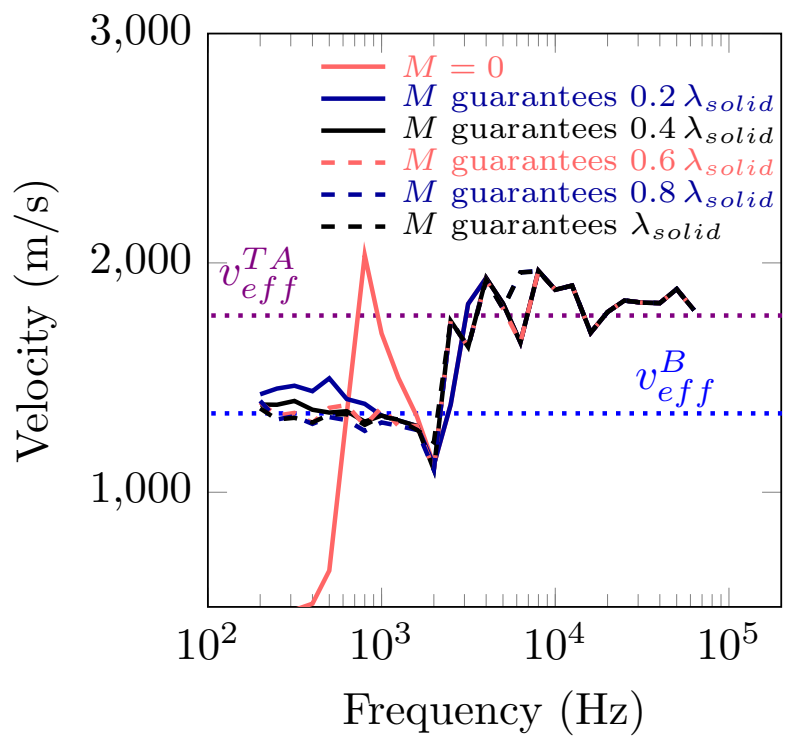

Figure 20: Effective velocities for the sample 2DVTI 12x12 (1F2S) for different values of $M$.

It is possible to repeat infinite times the rock in the perpendicular directions to the source. To do so, it is sufficient to mirror the original rock once in $2 \mathrm{D}$ (or four times in 3D) and impose periodic boundary conditions (see Figure 21). While this is a viable venue, we did not explore it in this work. Notice, however, that a similar alternative does not exist in the propagation direction due to the presence of the source.

\subsection{Experiment 11: VTI Rocks}

This experiment compares the solution for the VTI Rock 2DVTI 12x12 (1F2S) defined in Table 4 with the solution computed for the Rock 1DP12(1F2S). We post-process the solution over one vertical line located at the center of the rock.

Figure 22 shows similar results for $1 \mathrm{D}$ and 2D. However, in the case of a large contrast between the two densities, the 2D solution exhibits additional oscillations in the transition zone before reaching the high-frequency velocity limit.

Table 11 shows a match between the velocity computed with the FMM and with the Wyllie time-average formula (3). 


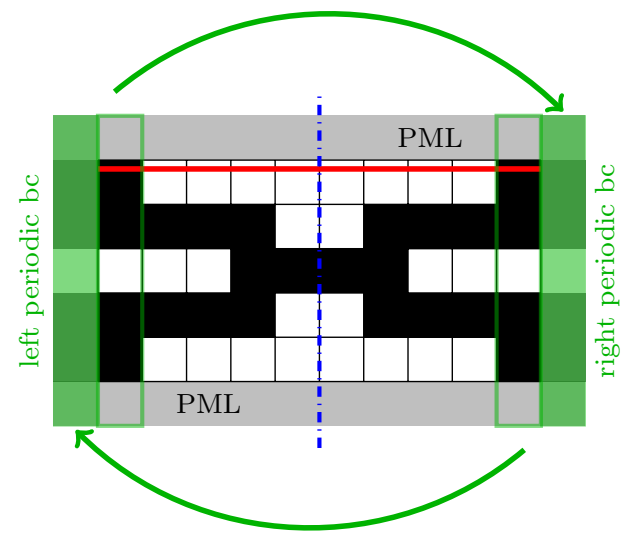

Figure 21: 2D model problem with periodic boundary conditions (BCs) in the direction perpendicular to the source (red line). The original rock is mirrored according to the axis of symmetry (blue line).

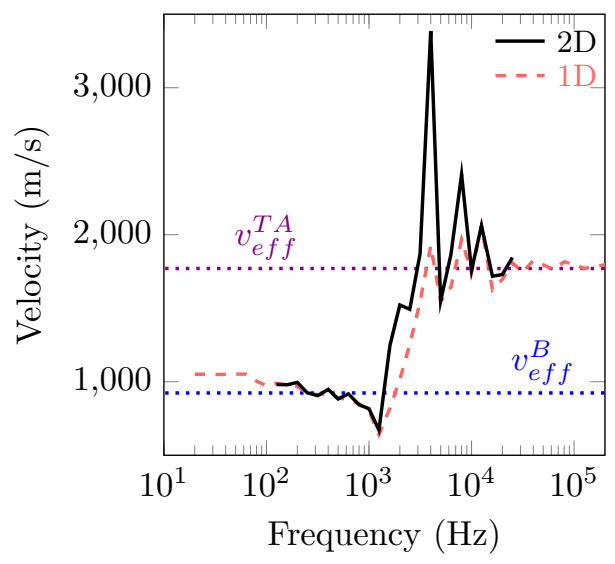

(a) $\rho_{S}=2800 \mathrm{~kg} / \mathrm{m}^{3}$

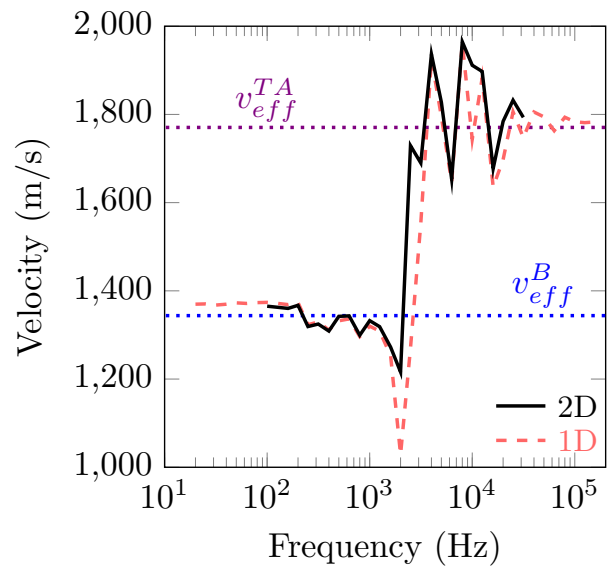

(b) $\rho_{S}=1000 \mathrm{~kg} / \mathrm{m}^{3}$

Figure 22: Comparative of the effective compressional wave velocity between a 2D 12x12 VTI (1F2S) rock and its 1D equivalent.

\subsection{Experiment 12: HTI Rocks}

Figure 23 shows the effective P-wave velocity for the periodic rock 2DHTI $12 \times 12(1 \mathrm{~F} 2 \mathrm{~T})$ defined in Table 4 . In this case, the choice of the analysis line is crucial for the accurate computation of the effective velocity.

The effective compressional wave velocity computed by FMM matches with the value of the fast material, regardless the porosity (see Table 12). On the 
Table 11: Effective P-wave velocities computed with Time-Average and FMM for 2D and 3D VTI rocks

\begin{tabular}{llll}
\hline Nomenclature & porosity & $v_{\text {eff }}^{\text {TA }}(\mathrm{m} / \mathrm{s})$ & FMM $(\mathrm{m} / \mathrm{s})$ \\
\hline${\text { 2D VTI }(1 \mathrm{~F} 2 \mathrm{~S})^{*}}^{*} 33.3 \%$ & 1770.49 & 1768.92 \\
2D VTI $(1 \mathrm{~F} 3 \mathrm{~S})^{*}$ & $25 \%$ & 2086.96 & 2084.77 \\
2D VTI $(1 \mathrm{~F} 7 \mathrm{~S})^{*}$ & $12.5 \%$ & 2541.18 & 2538.43 \\
${\text { 3D VTI }(1 \mathrm{~F} 3 \mathrm{~S})^{* *}}^{2} 25 \%$ & 2086.96 & 2086.96 \\
\hline
\end{tabular}

* Size of 1200x1200 pixels

** Size of $100 \times 100 \times 100$ voxels

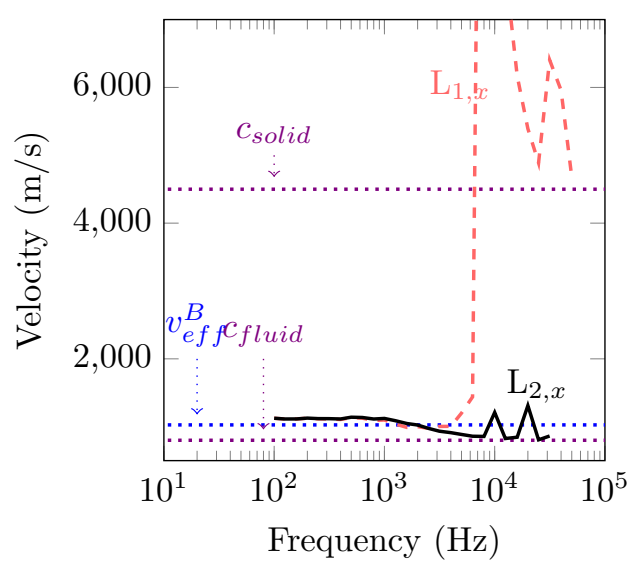

(a) $\rho_{S}=2800 \mathrm{~kg} / \mathrm{m}^{3}$

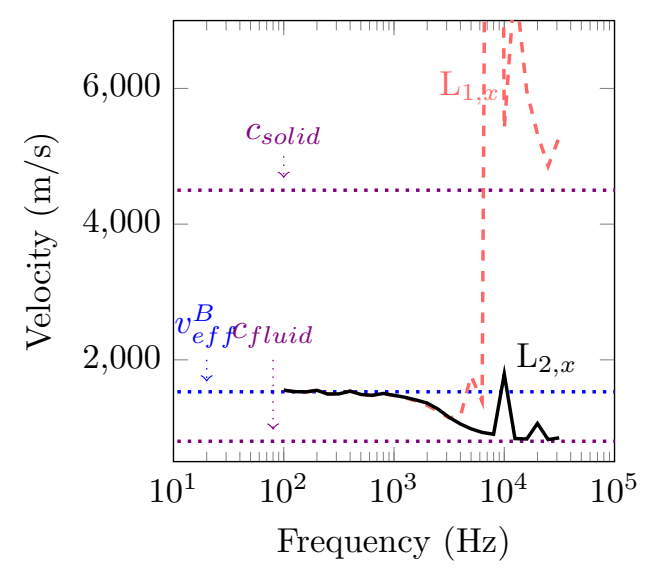

(b) $\rho_{S}=1000 \mathrm{~kg} / \mathrm{m}^{3}$

Figure 23: Effective compressional wave velocity for 2D HTI 12x12 (1F2S) rock. The black solid curve corresponds to the effective $\mathrm{P}$-wave velocity measured along the fluid $\left(\mathrm{L}_{2, x}\right)$, while the dashed red curve is recorded along the solid $\left(\mathrm{L}_{1, x}\right)$.

other hand, the velocity computed by the Wyllie time-average formula (3) is much lower. This result confirms that the use of the Wyllie time-average formula is invalid outside of VTI symmetries, as physically expected.

\subsection{Experiment 13: The Labyrinth}

In this experiment, we consider the labyrinth depicted in Figure 9 At high frequencies, the first arriving wave circumvents the fluid by following the solid red line in Figure 24a. Its effective velocity is approximately $c_{\text {solid }} / 2$.

Figure 24b displays the effective velocity computed with the FMM at the bottom of the domain as a function of space. These results confirm that the FMM is trustworthy and provides physically consistent velocities at all arriving points in a non-trivial domain. 


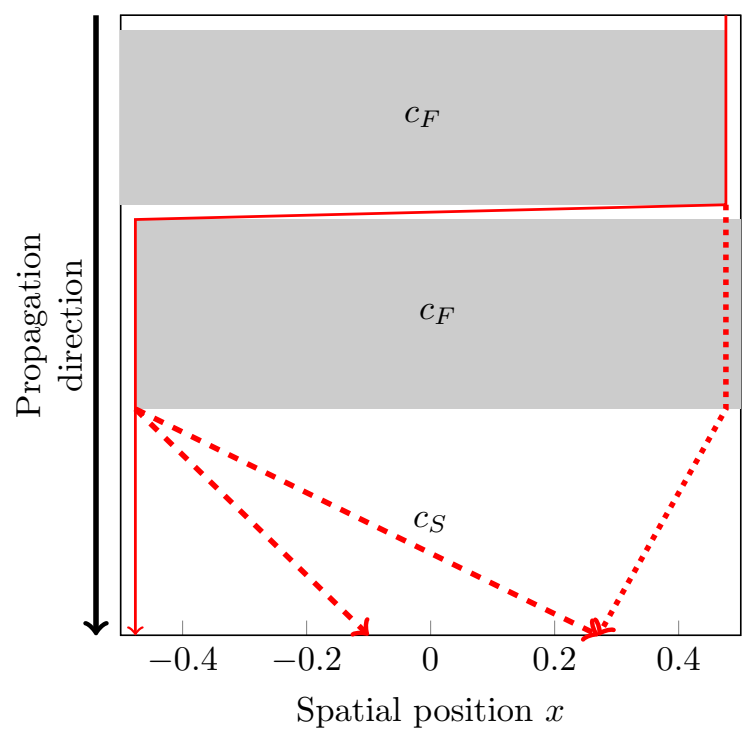

(a) Wave propagation paths

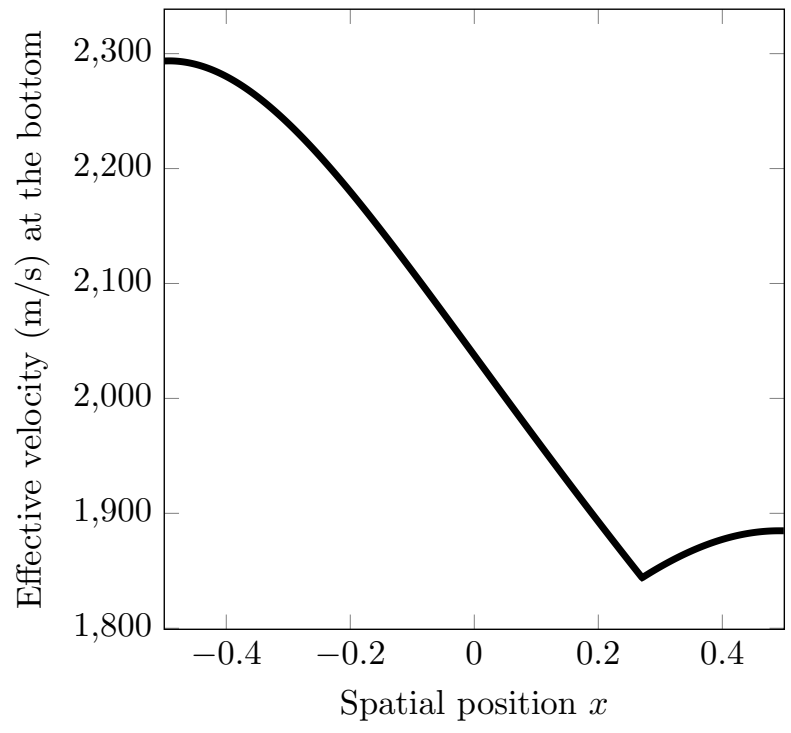

(b) Effective velocity at the bottom of the core

Figure 24: Sketch of the labyrinth domain and the computed effective velocity at the bottom of the core by the FMM. 
Table 12: Effective P-wave velocities computed with TimeAverage and FMM. $c_{\text {solid }}=4500 \mathrm{~m} / \mathrm{s}$

\begin{tabular}{llll}
\hline Nomenclature & porosity & $v_{\text {eff }}^{\text {TA }}(\mathrm{m} / \mathrm{s})$ & FMM $(\mathrm{m} / \mathrm{s})$ \\
\hline${\text { 2D HTI }(1 \mathrm{~F} 2 \mathrm{~S})^{*}}^{*}$ & $33.3 \%$ & 1770.49 & 4500.00 \\
2D HTI $(1 \mathrm{~F} 3 \mathrm{~S})^{*}$ & $25 \%$ & 2086.96 & 4500.00 \\
2D HTI $(1 \mathrm{~F} 7 \mathrm{~S})^{*}$ & $12.5 \%$ & 2541.18 & 4500.00 \\
3D HTI $_{x}(1 \mathrm{~F} 3 \mathrm{~S})^{* *}$ & $25 \%$ & 2086.96 & 4499.99 \\
3D HTI $_{y}(1 \mathrm{~F} 3 \mathrm{~S})^{* *}$ & $25 \%$ & 2086.96 & 4499.99 \\
\hline
\end{tabular}

* Size of $1200 \times 1200$ pixels

** Size of $100 \times 100 \times 100$ voxels

\subsection{Experiment 14: Real rocks}

Figures 25 and 26 show the effective $\mathrm{P}$-wave velocity average analyzed along five lines in a cross-section of 3D Rock1 (see Table 6), and 3D Rock 2, respectively.

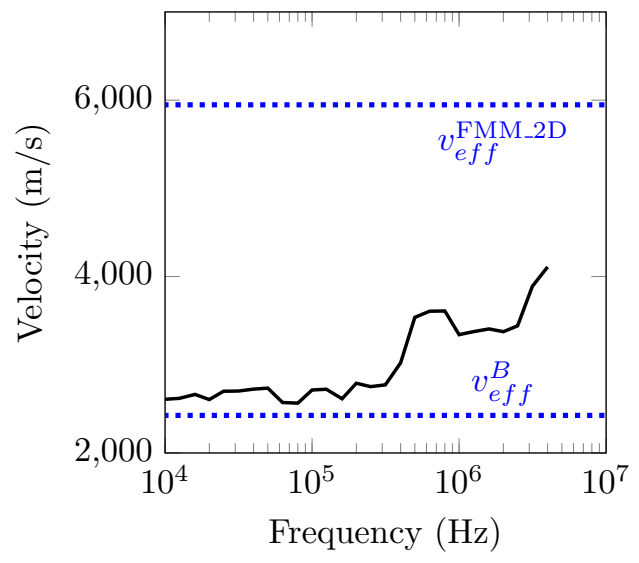

Figure 25: Average of effective P-wave velocity for a cross-section of 3DRock 1.

Table 13 collects the values computed with FMM for the full 3D rocks as well as some of their cross-sections. As expected, the effective compressional wave velocity computed in the high-frequency regime for the 3D rock is in between the one computed for the cross-section and the sound velocity of the fastest material. 


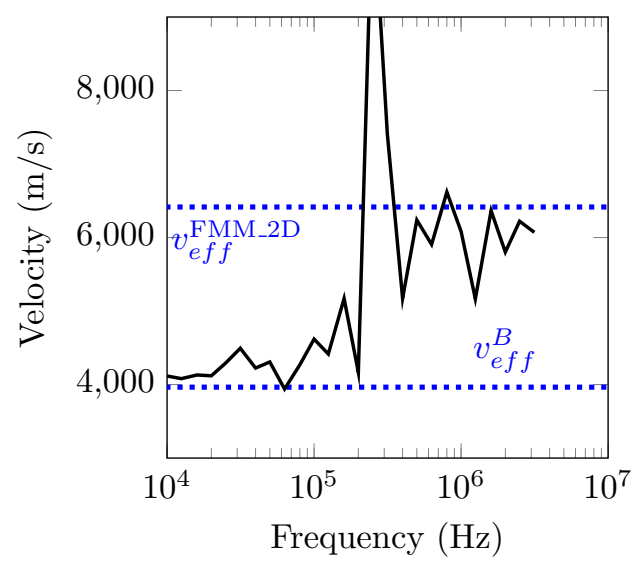

Figure 26: Average of effective P-wave velocity for a cross-section of 3DRock 2.

Table 13: Effective P-wave velocities computed by FMM and faster constituent sound velocity

\begin{tabular}{llll}
\hline Formation & $v_{\text {eff }}^{\mathrm{FMM} 2 \mathrm{D}}$ & $v_{\text {eff }}^{\mathrm{FMM}}(\mathrm{m} / \mathrm{s})$ & $c_{\text {fast }}(\mathrm{m} / \mathrm{s})$ \\
\hline 3D Rock 1 & 5946.94 & 6031.15 & 6041.98 \\
3D Rock 2 & 6415.495 & 6607.81 & 6645.11 \\
\hline
\end{tabular}

\section{Conclusions}

We propose a set of numerical methods to estimate the effective compressional wave velocity of heterogeneous rocks. At low frequencies, the PML pollutes the values of the computed effective compressional wave velocity. Thus, we extend the domain through rock repetition in order to guarantee at least two wavelengths in the propagation direction. To make the resulting problem computationally tractable, we employ non-fitting meshes, which introduce some controlled numerical errors. When carefully implemented, the resulting effective velocities exhibit acceptable accuracies. The Prony's post-processing method provides accurate values of the effective compressional wave velocity in the Backus average zone, but the method diverges in the transition zone, where the behavior of the wave is no longer given as a sum of plane waves. On the other hand, the value of the effective compressional wave velocity estimated by counting the numbers of wavelengths converges in the entire frequency spectrum.

At high frequencies, the computation of the effective velocity by FMM matches with the theoretical limit expected in VTI rocks. We show the validity of this numerical method in higher spatial dimensions.

Numerical results, in accordance with the existing theory, indicate the following: (a) an increase in the density of the fast material decreases the value of the effective compressional wave velocity at low frequencies. On the other 
hand, effective velocities at high frequencies become unaltered by these density changes, as predicted by the theory; (b) an increase in the porosity produces a decrease in the value of the effective compressional wave velocity. This decrease is more pronounced at high frequencies; (c) the presence of different sizes of the pore produces a widening of the transition zone since the most significant size of the pore is related to the frequency regime in which the Backus average is valid, while the smallest size of the pore characterizes the frequency for which the time average holds; (d) the Wyllie time-average formula fails outside of VTI rocks.

Possible extensions of this work include the use of parametric solvers like the one presented in [41] for the low frequency spectrum to analyze simultaneously a group of rocks, and multilevel methods [4] for the medium frequency spectrum.

\section{Acknowledgements}

This research was supported by Repsol, the European Union's Horizon 2020 research and innovation programme under the Marie Sklodowska-Curie grant agreement No 777778 (MATHROCKS), the European POCTEFA 20142020 Project PIXIL (EFA362/19) by the European Regional Development Fund (ERDF) through the Interreg V-A Spain-France-Andorra programme, the Project of the Spanish Ministry of Economy and Competitiveness with reference MTM-2016-76329-R (AEI/FEDER, EU), the BCAM "Severo Ochoa" accreditation of excellence (SEV-2017-0718), and the Basque Government through the BERC 2018-2021 program, the two Elkartek projects ArgIA (KK2019-00068) and MATHEO (KK-2019-00085), the grant "Artificial Intelligence in BCAM number EXP. 2019/00432", and the Consolidated Research Group MATHMODE (IT1294-19) given by the Department of Education.

\section{References}

[1] Abdulle, A., E, W., Engquist, B., Vanden-Eijnden, E.: The heterogeneous multiscale method. Acta Numerica 21, 1-87 (2012). DOI 10.1017/ S0962492912000025

[2] Aydin, A.: Upgraded ISRM suggested method for determining sound velocity by ultrasonic pulse transmission technique. Rock Mechanics and Rock Engineering 47(1), 255-259 (2014). DOI 10.1007/s00603-013-0454-z. URL https://doi.org/10.1007/s00603-013-0454-z

[3] Backus, G.: Long-wave elastic anisotropy produced by horizontal layering. Journal of Geophysical Research 67(11), 4427-4440 (1962). DOI 10.1029/ JZ067i011p04427. URL https://agupubs.onlinelibrary.wiley.com/ doi/abs/10.1029/JZ067i011p04427

[4] Berenger, J.: A perfectly matched layer for the absorption of electromagnetic waves. Journal of computational physics 114(2), 185-200 (1994) 
[5] Bergmann, P.G.: The wave equation in a medium with a variable index of refraction. The Journal of the Acoustical Society of America 17(4), 329333 (1946). DOI 10.1121/1.1916333. URL https://doi.org/10.1121/1. 1916333

[6] Berryman, J.G.: Long-wave elastic anisotropy in transversely isotropic media. GEOPHYSICS 44(5), 896-917 (1979). DOI 10.1190/1.1440984. URL https://doi.org/10.1190/1.1440984

[7] Berryman, J.G.: Origin of Gassmann's equations. GEOPHYSICS 64(5), 1627-1629 (1999). DOI 10.1190/1.1444667. URL https://doi.org/10. $1190 / 1.1444667$

[8] Biot, M.A.: Theory of propagation of elastic waves in a fluid-saturated porous solid. i. low-frequency range. The Journal of the Acoustical Society of America 28(2), 168-178 (1956). DOI 10.1121/1.1908239. URL https : //doi.org/10.1121/1.1908239

[9] Bonnasse-Gahot, M., Calandra, H., Diaz, J., Lanteri, S.: Hybridizable discontinuous Galerkin method for the 2D frequency-domain elastic wave equations. Geophysical Journal International 213(1), 637-659 (2017). DOI 10.1093/gji/ggx533. URL https://doi.org/10.1093/gji/ggx533

[10] Calo, V.M., Efendiev, Y., Galvis, J., Li, G.: Randomized oversampling for generalized multiscale finite element methods. Multiscale Modeling \& Simulation 14(1), 482-501 (2016). DOI 10.1137/140988826. URL https://doi.org/10.1137/140988826

[11] Cance, P., Capdeville, Y.: Validity of the acoustic approximation for elastic waves in heterogeneous media. GEOPHYSICS 80(4), T161-T173 (2015). DOI 10.1190/geo2014-0397.1. URL https://doi.org/10.1190/ geo2014-0397.1

[12] Carcione, J.: Wave fields in real media: Wave propagation in anisotropic, anelastic, porous and electromagnetic media, vol. 38. Elsevier (2007)

[13] Chapman, M.: Frequency-dependent anisotropy due to meso-scale fractures in the presence of equant porosity. Geophysical Prospecting 51(5), 369-379 (2003). DOI $10.1046 / \mathrm{j} .1365-2478.2003 .00384$. x. URL https://onlinelibrary.wiley.com/doi/abs/10.1046/j. 1365-2478.2003.00384.x

[14] Chaumont-Frelet, T., Nicaise, S., Pardo, D.: Finite element approximation of electromagnetic fields using nonfitting meshes for geophysics. SIAM Journal on Numerical Analysis 56(4), 2288-2321 (2018). DOI 10.1137/ 16M1105566. URL https://doi.org/10.1137/16M1105566

[15] Chaumont-Frelet, T., Valentin, F.: A multiscale hybrid-mixed method for the helmholtz equation in heterogeneous domains. SIAM Journal on 
Numerical Analysis 58(2), 1029-1067 (2020). DOI 10.1137/19M1255616. URL https://doi.org/10.1137/19M1255616

[16] Cheng, C.H.: Crack models for a transversely isotropic medium. Journal of Geophysical Research: Solid Earth 98(B1), 675-684 (1993). DOI 10.1029/92JB02118. URL https://agupubs . onlinelibrary.wiley.com/ doi/abs/10.1029/92JB02118

[17] Cioranescu, D., Donato, P.: An Introduction to Homogenization. Oxford lecture series in mathematics and its applications. Oxford University Press (1999)

[18] Ciz, R., Shapiro, S.A.: Generalization of Gassmann equations for porous media saturated with a solid material. GEOPHYSICS 72(6), A75-A79 (2007). DOI 10.1190/1.2772400. URL https://doi.org/10.1190/1. 2772400

[19] Cnudde, V., Boone, M.: High-resolution x-ray computed tomography in geosciences: A review of the current technology and applications. EarthScience Reviews 123, 1 - 17 (2013). DOI https://doi.org/10.1016/j. earscirev.2013.04.003. URL http://www.sciencedirect.com/science/ article/pii/S001282521300069X

[20] Dvorkin, J., Nur, A.: Time-average equation revisited. GEOPHYSICS 63(2), 460-464 (1998). DOI 10.1190/1.1444347. URL https://doi.org/ $10.1190 / 1.1444347$

[21] Efendiev, Y., Galvis, J., Li, G., Presho, M.: Generalized multiscale finite element methods: Oversampling strategies. International Journal for Multiscale Computational Engineering 12(6), 465-484 (2014). DOI 10.1615/IntJMultCompEng.2014007646

[22] Eshelby, J.D., Peierls, R.E.: The determination of the elastic field of an ellipsoidal inclusion, and related problems. Proceedings of the Royal Society of London. Series A. Mathematical and Physical Sciences 241(1226), 376-396 (1957). DOI 10.1098/rspa.1957.0133. URL https: //royalsocietypublishing.org/doi/abs/10.1098/rspa.1957.0133

[23] Francfort, G.A., Murat, F.: Homogenization and optimal bounds in linear elasticity. Archive for Rational Mechanics and Analysis 94(4), 307-334 (1986). DOI 10.1007/BF00280908. URL https://doi.org/10.1007/ BF00280908

[24] Gardner, G.H.F., Gardner, L.W., Gregory, A.R.: Formation velocity and density-the diagnostic basics for stratigraphic traps. GEOPHYSICS 39(6), 770-780 (1974). DOI 10.1190/1.1440465. URL https://doi.org/10. $1190 / 1.1440465$ 
[25] Gassmann, F.: Über die elastizität poröser medien (on elasticity of porous media). Veirteljahrsschrift der Naturforschenden Gesellschaft in Zürich 96, $1-23(1951)$

[26] Geers, M., Kouznetsova, V., Brekelmans, W.: Multi-scale computational homogenization: Trends and challenges. Journal of Computational and Applied Mathematics 234(7), 2175 - 2182 (2010). DOI https://doi. org/10.1016/j.cam.2009.08.077. URL http://www.sciencedirect.com/ science/article/pii/S0377042709005536. Fourth International Conference on Advanced COmputational Methods in ENgineering (ACOMEN 2008)

[27] Grechka, V., Kachanov, M.: Effective elasticity of rocks with closely spaced and intersecting cracks. GEOPHYSICS 71(3), D85-D91 (2006). DOI 10.1190/1.2197489. URL https://doi.org/10.1190/1.2197489

[28] Hashin, Z., Shtrikman, S.: On some variational principles in anisotropic and nonhomogeneous elasticity. Journal of the Mechanics and Physics of Solids 10(4), 335 - 342 (1962). DOI https://doi.org/10.1016/0022-5096(62) 90004-2. URL http://www.sciencedirect.com/science/article/pii/ 0022509662900042

[29] Hashin, Z., Shtrikman, S.: A variational approach to the theory of the elastic behaviour of multiphase materials. Journal of the Mechanics and Physics of Solids 11(2), 127 - 140 (1963). DOI https://doi.org/10.1016/ 0022-5096(63)90060-7. URL http://www.sciencedirect.com/science/ article/pii/0022509663900607

[30] Helbig, K.: Anisotropy and dispersion in periodically layered media. Geophysics 49(4), 364-373 (1984)

[31] Hill, R.: The elastic behaviour of a crystalline aggregate. Proceedings of the Physical Society. Section A 65(5), 349-354 (1952). DOI 10.1088/ 0370- $1298 / 65 / 5 / 307$

[32] Hovem, J.: Acoustic waves in finely layered media. Geophysics 60(4), 1217-1221 (1995). DOI 10.1190/1.1443850. URL https://doi.org/10. $1190 / 1.1443850$

[33] Hudson, J.A.: Wave speeds and attenuation of elastic waves in material containing cracks. Geophysical Journal International 64(1), 133-150 (1981). DOI 10.1111/j.1365-246X.1981.tb02662.x. URL https://dx.doi. org/10.1111/j.1365-246X.1981.tb02662.x

[34] Kuster, G.T., Toksöz, M.N.: Velocity and attenuation of seismic waves in two-phase media: Part i. theoretical formulations. GEOPHYSICS 39(5), 587-606 (1974). DOI 10.1190/1.1440450. URL https://doi.org/10. $1190 / 1.1440450$ 
[35] Levin, F.K.: Seismic velocities in transversely isotropic media. GEOPHYSICS 44(5), 918-936 (1979). DOI 10.1190/1.1440985. URL https: //doi.org/10.1190/1.1440985

[36] Marion, D., Coudin, P.: Fram ray to effective medium theories in stratified media: An experimental study. In: SEG Technical Program Expanded Abstracts 1992, pp. 1341-1343. Society of Exploration Geophysicists (1992)

[37] Marion, D., Mukerji, T., Mavko, G.: Scale effects on velocity dispersion: From ray to effective medium theories in stratified media. Geophysics 59(10), 1613-1619 (1994)

[38] Matouš, K., Geers, M.G., Kouznetsova, V.G., Gillman, A.: A review of predictive nonlinear theories for multiscale modeling of heterogeneous materials. Journal of Computational Physics 330, $192-$ 220 (2017). DOI https://doi.org/10.1016/j.jcp.2016.10.070. URL http: //www.sciencedirect.com/science/article/pii/S0021999116305782

[39] Matuszyk, P.J., Torres-Verdín, C., Pardo, D.: Frequency-domain finiteelement simulations of 2D sonic wireline borehole measurements acquired in fractured and thinly bedded formations. GEOPHYSICS 78(4), D193D207 (2013). DOI 10.1190/geo2012-0397.1. URL https://doi.org/10. 1190/geo2012-0397.1

[40] Mavko, G., Mukerji, T., Dvorkin, J.: The Rock Physics Handbook: Tools for Seismic Analysis of Porous Media, 2nd edn. Cambridge University Press, Cambridge (2009). URL http://www. worldcat.org/isbn/0521861365

[41] Modesto, D., Zlotnik, S., Huerta, A.: Proper generalized decomposition for parameterized helmholtz problems in heterogeneous and unbounded domains: Application to harbor agitation. Computer Methods in Applied Mechanics and Engineering 295, 127 - 149 (2015). DOI https://doi. org/10.1016/j.cma.2015.03.026. URL http://www.sciencedirect.com/ science/article/pii/S004578251500136X

[42] Müller, T.M., Caspari, E., Qi, Q., Rubino, J.G., Velis, D., Lopes, S., Lebedev, M., Gurevich, B.: Chapter 3 - acoustics of partially saturated rocks: Theory and experiments. In: J. Ba, Q. Du, J.M. Carcione, H.J. Zhang, T.M. Müller (eds.) Seismic Exploration of Hydrocarbons in Heterogeneous Reservoirs, pp. 45 - 75. Elsevier (2015). DOI https://doi.org/10.1016/B978-0-12-420151-4.00003-9. URL http://www. sciencedirect.com/science/article/pii/B9780124201514000039

[43] Norris, A.: A differential scheme for the effective moduli of composites. Mechanics of Materials 4(1), $1-16$ (1985). DOI https://doi.org/10.1016/ 0167-6636(85)90002-X. URL http://www.sciencedirect.com/science/ article/pii/016766368590002X 
[44] Oleinik, O., Shamaev, A., Yosifian, G.: Mathematical Problems in Elasticity and Homogenization. ISSN. Elsevier Science (2009)

[45] Osborne, M., Smyth, G.: A modified prony algorithm for exponential function fitting. SIAM Journal on Scientific Computing 16(1), 119-138 (1995)

[46] Prony, C., Gaspard, F., De-Baron, R.: Essai experimental et analytique. Journal de l'Ecole Polytechnique de Paris 1, 24-76 (1795)

[47] Rachowicz, W., Zdunek, A.: Automated multi-level substructuring (amls) for electromagnetics. Computer Methods in Applied Mechanics and Engineering 198(13), 1224 - 1234 (2009). DOI https://doi. org/10.1016/j.cma.2008.10.011. URL http://www.sciencedirect.com/ science/article/pii/S0045782508003605. HOFEM07

[48] Rawlinson, N., Hauser, J., M., S.: Seismic ray tracing and wavefront tracking in laterally heterogeneous media. Advances in Geophysics 49, 203-273 (2008). DOI https://doi.org/10.1016/S0065-2687(07)49003-3

[49] Raymer, L.L., Hunt, E.R., Gardner, J.S.: An improved sonic transit timeto-porosity transform. Society of Petrophysicists and Well-Log Analysts pp. 1-12 (1980)

[50] Reuss, A.: Berechnung der fließgrenze von mischkristallen auf grund der plastizitätsbedingung für einkristalle . ZAMM - Journal of Applied Mathematics and Mechanics / Zeitschrift für Angewandte Mathematik und Mechanik 9(1), 49-58 (1929). DOI 10.1002/zamm.19290090104

[51] Romkes, A., Oden, J.T.: Adaptive modeling of wave propagation in heterogeneous elastic solids. Computer Methods in Applied Mechanics and Engineering 193(6-8), 539-559 (2004). DOI 10.1016/j.cma.2003.10.014

[52] Rouy, E., Tourin, A.: A viscosity solutions approach to shape-from-shading. SIAM J. Num. Anal. 29, 867-884 (1992). DOI https://doi.org/10.1137/ 0729053

[53] Saleh, A.A., Castagna, J.P.: Revisiting the wyllie time average equation in the case of near-spherical pores. GEOPHYSICS 69(1), 45-55 (2004). DOI 10.1190/1.1649374. URL https://doi.org/10.1190/1.1649374

[54] Schoenberg, M., Muir, F.: A calculus for finely layered anisotropic media. GEOPHYSICS 54(5), 581-589 (1989). DOI 10.1190/1.1442685. URL https://doi.org/10.1190/1.1442685

[55] Schot, S.H.: Eighty years of sommerfeld's radiation condition. Historia Mathematica 19(4), 385 - 401 (1992). DOI https://doi.org/10.1016/ 0315-0860(92)90004-U. URL http://www. sciencedirect.com/science/ article/pii/031508609290004U 
[56] Sethian, J.A.: Evolution, implementation, and application of level set and fast marching methods for advancing fronts. J. Comp. Phys. 169, 503-555 (2001). DOI https://doi.org/10.1006/jcph.2000.6657

[57] Sethian, J.A., Popovici, A.M.: 3-d traveltime computation using the fast marching method. Geophysics 64, 516-523 (1999). DOI https://doi.org/ $10.1190 / 1.1444558$

[58] Sommerfeld, A.: Die greensche funktion der schwingungslgleichung. Jahresbericht der Deutschen Mathematiker-Vereinigung 21, 309 - 352 (1912). URL http://eudml.org/doc/145344

[59] Stovas, A., Arntsen, B.: Low frequency waves in finely layered media. In: 65th EAGE Conference \& Exhibition (2003)

[60] Stovas, A., Ursin, B.: Equivalent time-average and effective medium for periodic layers. Geophysical Prospecting 55(6), 871-882 (2007). DOI 10.1111/j.1365-2478.2007.00653.x. URL https://onlinelibrary.wiley. com/doi/abs/10.1111/j.1365-2478.2007.00653.x

[61] Thomsen, L.: Elastic anisotropy due to aligned cracks in porous rock1. Geophysical Prospecting 43(6), 805-829 (1995). DOI 10.1111/j.1365-2478. 1995.tb00282.x. URL https://onlinelibrary.wiley.com/doi/abs/10. 1111/j.1365-2478.1995.tb00282.x

[62] Voigt, W.: Ueber die beziehung zwischen den beiden elasticitätsconstanten isotroper körper. Annalen der Physik 274(12), 573-587 (1889). DOI 10.1002/andp.18892741206

[63] Wyllie, M.R.J., Gregory, A.R., Gardner, L.W.: Elastic wave velocities in heterogeneous and porous media. GEOPHYSICS 21(1), 41-70 (1956). DOI 10.1190/1.1438217. URL https://doi.org/10.1190/1.1438217 